\title{
Electroweakino constraints from LHC data
}

\author{
Travis A.W. Martin and David E. Morrissey \\ TRIUMF, \\ 4004 Wesbrook Mall, Vancouver, V6T $2 A 3$ Canada \\ E-mail: tmartin@triumf.ca, dmorri@triumf.ca
}

ABSTRACT: We investigate the sensitivity of existing LHC searches to the charginos and neutralinos of the MSSM when all the other superpartners are decoupled. In this limit, the underlying parameter space reduces to a simple four-dimensional set $\left\{M_{1}, M_{2}, \mu, \tan \beta\right\}$. We examine the constraints placed on this parameter space by a broad range of LHC searches taking into account the full set of relevant production and decay channels. We find that the exclusions implied by these searches exceed existing limits from LEP only for smaller values of the Bino mass $M_{1} \lesssim 150 \mathrm{GeV}$. Our results have implications for MSSM dark matter and electroweak baryogenesis.

Keywords: Supersymmetry Phenomenology, Hadronic Colliders

ARXiv EPRINT: 1409.6322 


\section{Contents}

1 Introduction 1

2 Masses and mixings, production and decay 2

2.1 LHC production rates 3

$\begin{array}{ll}2.2 & \text { Decay branching fractions }\end{array}$

$\begin{array}{ll}2.3 & \text { Implications for LHC signals } \\ \end{array}$

3 Methodology of LHC sensitivity estimates 11

4 Limits from the LHC

$\begin{array}{lll}4.1 & \text { ATLAS opposite sign dileptons } & 13\end{array}$

$\begin{array}{lll}4.2 & \text { ATLAS trilepton } & 14\end{array}$

$\begin{array}{lll}4.3 & \text { ATLAS four lepton } & 15\end{array}$

$\begin{array}{lll}\text { 4.4 CMS lepton plus bottom quarks } & 16\end{array}$

$\begin{array}{lll}4.5 & \text { Other searches } & 17\end{array}$

$\begin{array}{ll}4.6 \text { Combined exclusions } & 17\end{array}$

5 Conclusions $\quad 18$

$\begin{array}{lr}\text { A Mass matrices and mixings } & 20\end{array}$

B Couplings to the standard model $\quad 21$

B.1 Vector boson couplings 21

B.2 (SM-like) Higgs couplings 22

\section{Introduction}

Supersymmetry (SUSY) is a well-motivated possibility for new physics, and is one of the main discovery targets of the Large Hadron Collider (LHC). A broad range of SUSY searches have been performed by the ATLAS and CMS collaborations with up to $5 \mathrm{fb}^{-1}$ of data at $7 \mathrm{TeV}$ and $20 \mathrm{fb}^{-1}$ of data at $8 \mathrm{TeV}$. Despite this great effort, no conclusive signals beyond the predictions of the Standard Model (SM) have been observed so far.

The absence of new signals puts constraints on the masses of the SM superpartners. The strongest bounds apply to the light-flavour squarks and the gluino, and can be as large as $m_{\tilde{q} / \tilde{g}} \gtrsim 1500 \mathrm{GeV}[1,2]$. Limits on stops and sbottoms, which must not be too heavy if they are to protect the naturalness of the weak scale [3], range between $m_{\tilde{t} / \tilde{b}} \gtrsim 200-700 \mathrm{GeV}$ depending on how they decay [4-8]. In contrast, superpartners that are uncharged under QCD can often be much lighter while remaining consistent with the current data. 
In the present work we study the implications of existing ATLAS and CMS searches on the charginos and neutralinos of the minimal supersymmetric standard model (MSSM). These states, which we will refer to collectively as electroweakinos, are mixtures of the superpartners of the electroweak vector and Higgs bosons, and they take the form of four Majorana-fermion neutralinos $\chi_{i}^{0}\left(i=1-4\right.$ with $\left.\left|m_{i}\right| \leq\left|m_{i+1}\right|\right)$ and two Dirac-fermion charginos $\chi_{i}^{ \pm}\left(i=1,2\right.$ with $\left.\left|m_{1}\right| \leq\left|m_{2}\right|\right)$. All other superpartners, namely the sfermions and the gluino, are assumed to be heavy enough that they can be neglected. Such a spectrum is motivated by the non-observation of squarks or the gluino, and can occur in theories of natural [9-13], or (mini-)split [14-22] supersymmetry. This leads to a relatively simple parameter space of four variables: $\left\{M_{1}, M_{2}, \mu, \tan \beta\right\}$.

A number of dedicated searches for electroweakinos have been performed by the LHC collaborations [23-25]. These searches focus primarily on final states with multiple leptons, and their results have been interpreted mainly within the context of simplified models $[26,27]$. Our work extends these results in three important ways. First, while simplified models are very useful in modelling key features of the production and decay processes, they do not capture the full dynamics of the MSSM. For example, multiple production channels can contribute importantly to the signal, and individual states can have many significant decay paths [28-31]. Second, we investigate the sensitivity to electroweakinos of a much broader range of searches than were considered by the ATLAS and CMS collaborations in this context. And third, we translate the search results into exclusions on $\left\{M_{1}, M_{2}, \mu, \tan \beta\right\}$, which has only recently been attempted in a limited way by the collaborations. This is useful for comparing with indirect limits on the electroweakinos, such as from flavour mixing and CP violation [32], precision electroweak tests [33], Higgs production and decay rates [34, 35], and cosmological processes like electroweak baryogenesis [36, 37] and dark matter production [38-40].

The implications of LHC searches on electroweakinos have also been the subject of many recent theoretical studies. These analyses often concentrate on specific collider topologies [41-43] or kinematic regimes [44-52], or are focussed on specific dark-mattermotivated scenarios [53-60]. Relative to these studies, we attempt to cover the MSSM parameter space more broadly, and without imposing any restrictions motivated by cosmology. At the same time, our analysis is more focussed on the electroweakinos than the detailed MSSM parameter scans considered in refs. [61, 62].

The outline of this paper is as follows. After this introduction, we describe the parameter ranges to be studied, their relationship to the spectrum, and their effect on production and decay processes in section 2. In section 3 we describe in detail the methods used to reinterpret the LHC results. Next, in section 4 we give our results in terms of exclusions on the underlying electroweakino parameters. Finally, section 5 is reserved for our conclusions. Formulas for the relevant masses and couplings are collected in appendices A and B.

\section{Masses and mixings, production and decay}

In this work we study the electroweakinos of the MSSM in the limit where all other superpartners (and the additional Higgs bosons) are much heavier. To be concrete, we set 
the values of the sfermion and gluino soft mass parameters to $2000 \mathrm{GeV}$ together with $m_{A}=1500 \mathrm{GeV}$, which effectively decouples these states from the LHC searches to be studied. This leaves a four-dimensional parameter space for the electroweakinos consisting of $\left\{M_{1}, M_{2}, \mu, \tan \beta\right\}$. We take as input the running values of these parameters at the scale $M_{S}=2000 \mathrm{GeV}$ and fix $\tan \beta=10 .{ }^{1}$ Explicit tree-level expressions for the electroweakino masses and couplings in terms of these parameters are collected in appendices A and B.

In most of the regions of interest, the diagonal mass-matrix elements, set by $M_{1}$ and $M_{2}$ for the gauginos and $\mu$ for the Higgsinos, are significantly larger than the off-diagonal elements, which are proportional to $m_{Z}$. As a result, the mass eigenstates tend to be closely aligned with the underlying gauge eigenstates unless there is a degeneracy among the diagonal terms. We will therefore speak frequently of Bino-like, Wino-like, and Higgsinolike mass eigenstates. The two Higgsino-like neutralino states coincide with the linear combinations

$$
\widetilde{H}_{ \pm}^{0}=\frac{1}{\sqrt{2}}\left(\widetilde{H}_{u}^{0} \pm \widetilde{H}_{d}^{0}\right)
$$

Away from degeneracies, the mixing of the Higgsinos $\widetilde{H}_{ \pm}^{0}$ with the gaugino $\widetilde{\lambda}_{a}(a=1,2)$ is proportional to $m_{Z} /\left|\mu \pm M_{a}\right|$, where $M_{a}$ is the relevant gaugino mass. Note that when $\mu$ and $M_{a}$ have the same sign, the $\widetilde{H}_{-}^{0}$ state mixes more strongly with the gaugino than the $\widetilde{H}_{+}^{0}[38]$. Mixing between different gaugino-like states requires two small mixing factors and is further suppressed. We will apply these considerations below to explain the relative production and decay rates of the physical chargino and neutralino states.

The relatively small mixing away from degeneracies also motivates us to focus on a specific value of $\tan \beta=10$. This parameter only enters into the properties of the electroweakinos through the off-diagonal elements of the mixing matrices (and in the direct couplings to the Higgs boson), as described in appendices A and B. Thus, we expect qualitatively similar results for production rates and decay fractions throughout the range $2 \leq \tan \beta \leq 50$ except where the splitting between two of $M_{1}, M_{2}$, or $\mu$ becomes small.

\subsection{LHC production rates}

In figure 1 we show the LHC8 production cross-sections of Bino-like, Wino-like, and Higgsino-like states for $\tan \beta=10$ with all other parameters taken to be much larger: $m=2000 \mathrm{GeV}$ for the sfermions and gluino and $m=1000 \mathrm{GeV}$ for the other electroweakino parameters. The physical masses in this limit are given approximately by $M_{1}, M_{2}$, or $\mu$. The neutral Higgsino-like states are labelled in order of increasing mass and correspond to the linear combinations $\widetilde{H}_{1}^{0} \sim \widetilde{H}_{-}^{0}$ and $\widetilde{H}_{2}^{0} \sim \widetilde{H}_{+}^{0}$ defined above. All rates shown in the figure are computed at leading order (LO) with MadGraph 5 [63] and cross-checked in Prospino2.1 [64, 65].

These production cross sections are dominated by processes with intermediate electroweak vector bosons. Both the Winos and Higgsinos couple to vector bosons through their gauge-covariant derivatives. This leads to unsuppressed couplings for $\chi_{i}^{0} \chi_{j}^{ \pm} W^{\mp}$ and

\footnotetext{
${ }^{1}$ The running of these parameters below this scale is mild, and we find nearly identical results using the same input values defined instead at $M_{S}=300 \mathrm{GeV}$.
} 


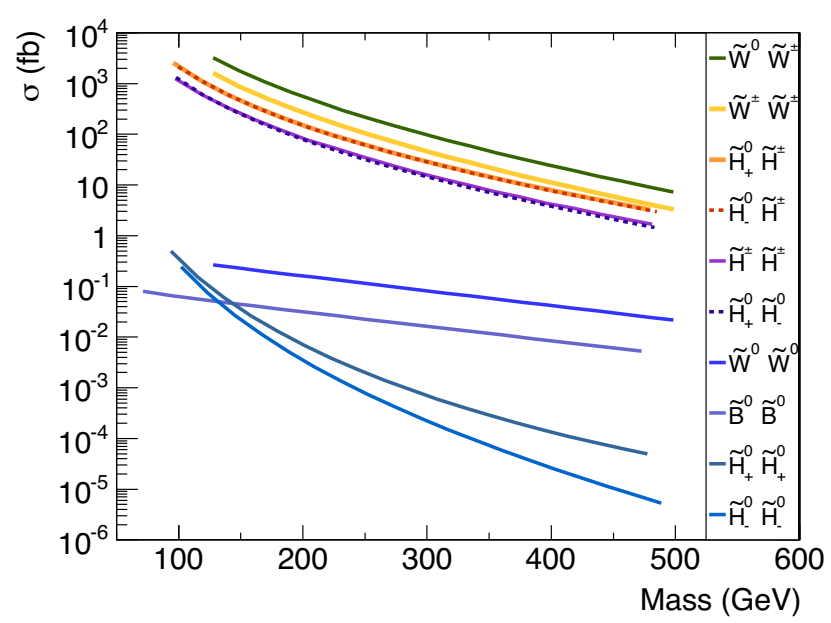

Figure 1. Production cross sections of the electroweakinos at the LHC with $\sqrt{s}=8 \mathrm{TeV}$ in the limit of nearly pure gauge eigenstates. One of $M_{1}, M_{2}$, or $\mu$ is varied independently with the other values set to $1000 \mathrm{TeV}$ and all other MSSM parameters set to $2000 \mathrm{GeV}$. The "Mass" label refers to the average mass of the two states being produced. Higgsino states are expressed in terms of $\widetilde{H}_{ \pm}^{0}=\left(\widetilde{H}_{u}^{0} \pm \widetilde{H}_{d}^{0}\right) / \sqrt{(2)}$.

$\chi_{i}^{ \pm} \chi_{j}^{\mp} Z^{0}$ when both states are pure Wino or pure Higgsino. In contrast, the neutralino couplings $\chi_{i}^{0} \chi_{j}^{0} Z^{0}$ involve only the Higgsino states, and are also suppressed when the $i=j$ state is Higgsino-like. The absence of a direct Wino coupling to the $Z^{0}$ arises because $\widetilde{W}^{0}$ has $t^{3}=Y=0$. Thus, the production rates of $\widetilde{W^{0}} \widetilde{W}^{0}$ and $\widetilde{B}^{0} \widetilde{B}^{0}$ are suppressed since both processes require two Higgsino mixings in the amplitude. The very small $\widetilde{H}_{i}^{0} \widetilde{H}_{i}^{0}$ rates are due to a cancellation in the pure Higgsino limit reflecting the fact that the corresponding mass eigenstates approach Dirac states with only a vector coupling to the $Z^{0}$ as $m_{Z} / \mu \rightarrow 0$. Production through a $W^{ \pm}$is generally larger than via the neutral vector bosons.

In figure 2 we show the dominant chargino and neutralino production cross sections as a function of the $\mu$ parameter for $\tan \beta=10$ and fixed $\left(M_{1}, M_{2}\right)=(200,300) \mathrm{GeV}$. Similar plots for $\left(M_{1}, M_{2}\right)=(300,200) \mathrm{GeV}$ are shown in figure 3 . These figures can be understood in terms of the gauge-eigenstate content of the corresponding mass eigenstates within the six possible hierarchies of $M_{1}, M_{2}$, and $\mu$.

Of the processes shown in figures 2 and 3, the chargino pair production rates in the leftmost panels are the easiest to understand. Here, the $\chi_{1}^{ \pm}$state is Higgsino-like for $\mu<M_{2}$ and evolves smoothly into a Wino-like state as $\mu$ increases above $M_{2}$. The $\chi_{1}^{+} \chi_{1}^{-}$and $\chi_{2}^{+} \chi_{2}^{-}$ rates follow the expectations for pure states in the appropriate limits, while the rate for $\chi_{1}^{ \pm} \chi_{2}^{\mp}$ is suppressed by the mixing factor it requires.

Neutralino-neutralino production, shown in the middle panels of figures 2 and 3, has a slightly more complicated dependence on $\mu$. Away from $\mu \sim M_{1}, M_{2}$, the physical mass eigenstates are closely aligned with pure gaugino $\left(\widetilde{W}^{0}, \widetilde{B}^{0}\right)$ or Higgsino $\left(\widetilde{H}_{ \pm}^{0}\right)$ states. The hierarchy of production rates can be understood by recalling that neutralino pair production occurs only through the $Z^{0} \widetilde{H}_{+}^{0} \widetilde{H}_{-}^{0}$ coupling, and that the $\widetilde{H}_{-}^{0}$-like state mixes much more readily with gauginos than the $\widetilde{H}_{+}^{0}$-like state (for $\mu$ and $M_{1,2}$ of the same sign). Thus, the 

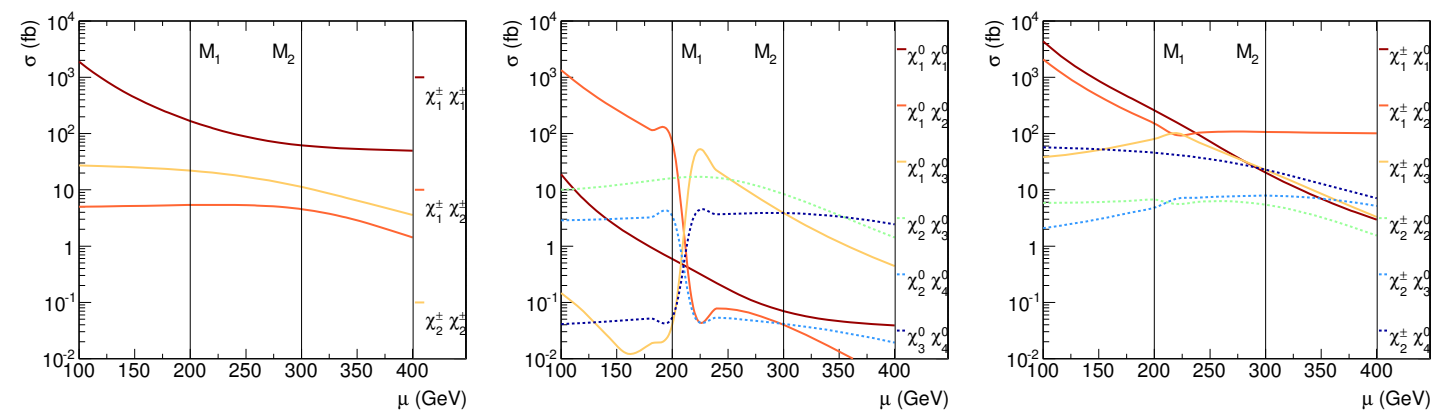

Figure 2. Leading electroweakino production cross sections as a function of $\mu$ for $M_{1}=200 \mathrm{GeV}$ and $M_{2}=300 \mathrm{GeV}$. The leftmost panel shows the chargino-chargino rates, the middle panel shows the dominant neutralino-neutralino rates, and the rightmost panel shows the largest charginoneutralino rates.
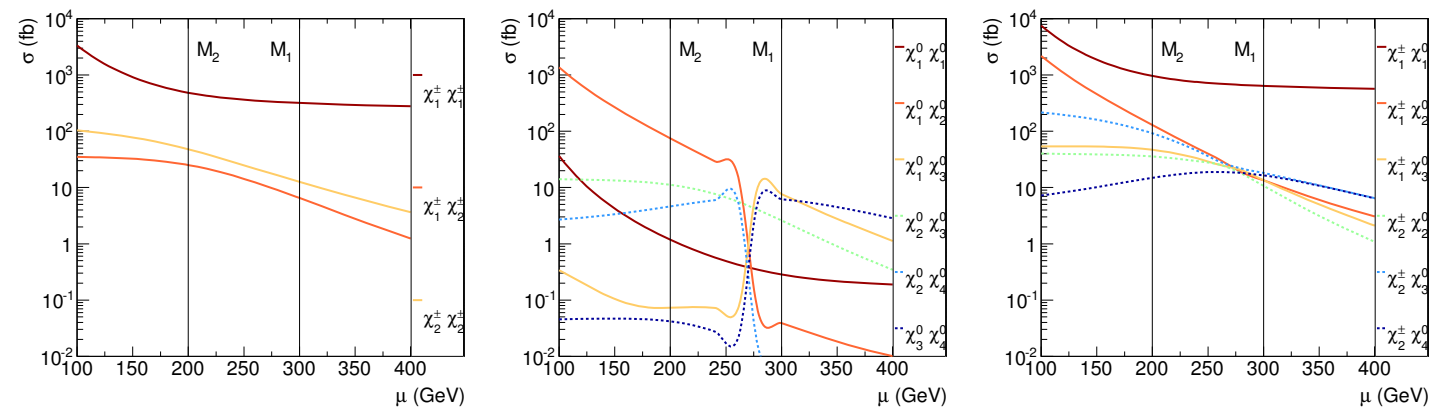

Figure 3. Leading electroweakino production cross sections as a function of $\mu$ for $M_{1}=200 \mathrm{GeV}$ and $M_{2}=300 \mathrm{GeV}$. The leftmost panel shows the chargino-chargino rates, the middle panel shows the dominant neutralino-neutralino rates, and the rightmost panel shows the largest charginoneutralino rates.

largest production rate occurs for the pair of states coinciding with $\widetilde{H}_{+}^{0} \widetilde{H}_{-}^{0}$, followed by gaugino- $\widetilde{H}_{+}^{0}$ pairs, and then gaugino- $\widetilde{H}_{-}^{0}$. The production of gaugino-gaugino or $\widetilde{H}_{ \pm}^{0} \widetilde{H}_{ \pm}^{0}$ pairs requires more small mixing factors and is further supressed.

These considerations explain the $\mu$ dependence of neutralino pair production seen in the middle panels of figures 2 and 3. The $\widetilde{H}_{+}^{0} \widetilde{H}_{-}^{0}$-like combination is $\chi_{1}^{0} \chi_{2}^{0}$ for $\mu<M_{<} \equiv$ $\min \left\{M_{1}, M_{2}\right\}, \chi_{2}^{0} \chi_{3}^{0}$ for $M_{<}<\mu<M_{>} \equiv \max \left\{M_{1}, M_{2}\right\}$, and $\chi_{3}^{0} \chi_{4}^{0}$ for $\mu>M_{>}$, and these are seen to have the largest rates (away from the gaugino masses). A sharp crossover is seen in both panels for the rates of $\chi_{1}^{0} \chi_{2}^{0}$ and $\chi_{1}^{0} \chi_{3}^{0}$ at a value of $\mu$ between $M_{<}$and $M_{>}$. For increasing $\mu$ in this range, mixing with the lighter gaugino tends to push the $\widetilde{H}_{-}^{0}$ mass up relative to $\widetilde{H}_{+}^{0}$, while mixing with the heavier gaugino tends to push the $\widetilde{H}_{-}^{0}$ mass down. This leads to a crossover where the $\widetilde{H}_{-}^{0}$-like state becomes lighter than the $\widetilde{H}_{+}^{0}$-like state, and the gauge contents of the mass-ordered $\chi_{2}^{0}$ and $\chi_{3}^{0}$ states are suddenly exchanged with each other. At this point, $\chi_{1}^{0} \chi_{2}^{0}$ goes from a moderately suppressed gaugino- $\widetilde{H}_{+}^{0}$ process to a highly suppressed gaugino- $\widetilde{H}_{-}^{0}$ process, with the opposite occurring for $\chi_{1}^{0} \chi_{3}^{0}$. A similar crossover is seen for the $\chi_{2}^{0} \chi_{4}^{0}$ and $\chi_{3}^{0} \chi_{4}^{0}$ rates. In both cases, the physically relevant quantity is the inclusive neutralino pair production rate, and this varies smoothly with $\mu$. 
The rightmost panels of figures 2 and 3 show the leading mixed neutralino-chargino production rates. For $\mu<M_{2}$, the largest cross sections occur for pairs of Higgsino-like states, such as $\chi_{1}^{ \pm} \chi_{1}^{0}$. As $\mu$ grows larger than $M_{2}$, there is a smooth transition such that the largest rates occur for pairs of Wino-like states. This corresponds to $\chi_{1}^{ \pm} \chi_{2}^{0}$ for $M_{1}<M_{2}$ and $\chi_{1}^{ \pm} \chi_{1}^{0}$ for $M_{1}>M_{2}$.

We have also examined the effects of varying $\tan \beta$ over the range $2 \leq \tan \beta \leq 50$. The dependence of the production cross sections on $\tan \beta$ saturates at larger values, with almost no variation between $\tan \beta=10$ and $\tan \beta=50$. For smaller $\tan \beta \sim 2$, the variation can be stronger near a mass degeneracy although the net qualitative effect tends to be mild.

\subsection{Decay branching fractions}

The collider signatures of the electroweakinos depend crucially on how they decay. When all the sfermions are very heavy, the dominant decay channels are

$$
\begin{array}{ccc}
\chi_{i}^{0} \rightarrow \chi_{j}^{ \pm} W^{\mp(*)}, & \chi_{i}^{0} \rightarrow \chi_{j}^{0} Z^{0(*)}, & \chi_{i}^{0} \rightarrow \chi_{j}^{0} h^{0(*)}, \\
\chi_{i}^{ \pm} \rightarrow \chi_{j}^{0} W^{ \pm(*)}, & \chi_{i}^{ \pm} \rightarrow \chi_{j}^{ \pm} Z^{0(*)}, & \chi_{i}^{ \pm} \rightarrow \chi_{j}^{ \pm} h^{0(*)},
\end{array}
$$

where $j<i$, and the $W^{ \pm}, Z^{0}$, and $h^{0}$ can be potentially off-shell (as indicated by $(*)$ ). A loop-mediated decay with a photon is also possible, but we almost always find it to be highly suppressed compared to the channels listed above.

The branching ratios of these decays depend on the gauge-eigenstate content and the mass splittings among the states. In figures $4-9$ we show the dominant gauge eigenstate components and the leading decay modes for all the neutralino and chargino states in the $\mu-M_{2}$ plane at fixed slices of $M_{1}=20,100,180,260,340 \mathrm{GeV}$ with $\tan \beta=10$. In all cases, the mixing factors and branching ratios were computed with SUSY-HIT 1.3 [66] interfaced with SuSpect 2.41 [67] or SoftSusy 3.3.10 [68]. The upper panels in figures 4-9 indicate where the dominant neutralino gauge component is $\widetilde{H}_{+}^{0}$ (light yellow), $\widetilde{H}_{-}^{0}$ (light-medium blue), $\widetilde{W}^{0}$ (dark-medium orange), or $\widetilde{B}^{0}$ (dark blue). The variations in shading in these panels show where the fraction of the corresponding state exceeds $50 \%$ or $75 \%$. The lower panels of figures 6-9 show the dominant decay fractions. The dotted, dot-dashed, and dashed lines in these figures indicate boundaries where the decay modes can occur on shell. While we only show results for positive values of $\mu, M_{1}$, and $M_{2}$, we find similar results for other relative signs.

The relative importance of the decay channels shown in figures 5-9 can be understood by counting the number of mixings required for each to occur while also taking into account the mass splitting between the initial and final states. Recall that the mixing goes like $m_{Z} /\left|M_{a} \pm \mu\right|$. As listed in appendix B, couplings to $W^{ \pm}$involve Wino-Wino or HiggsinoHiggsino, couplings to $Z^{0}$ involve only Higgsino-Higgsino, and couplings to $h^{0}$ involve Higgsino-Wino or Higgsino-Bino. The mass matrices of appendix A also show that the mass splitting between two relatively pure Wino-like or Higgsino-like states is less than about $m_{Z}$.

To illustrate this counting, and an additional subtlety associated with it, consider the decay of a Bino-like neutralino into a much lighter Wino-like neutralino or chargino. The gauge modes $\widetilde{B}^{0} \rightarrow W^{ \pm} \widetilde{W}^{\mp}$ and $\widetilde{B}^{0} \rightarrow Z^{0} \widetilde{W}^{0}$ both require two mixings in the decay 

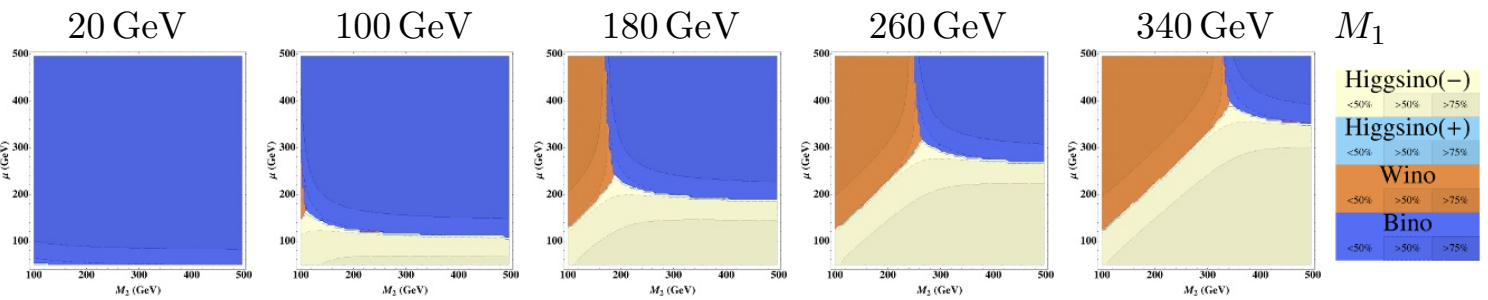

Figure 4. $\chi_{1}^{\mathbf{0}}$ : Dominant gauge eigenstate content of the lightest neutralino $\chi_{1}^{0}$ in the $M_{2}-\mu$ plane for various slices of $M_{1}$ and $\tan \beta=10$. Shaded, dash-enclosed regions indicate the boundary of $50 \%$ and $75 \%$ composition, as noted in the legend.
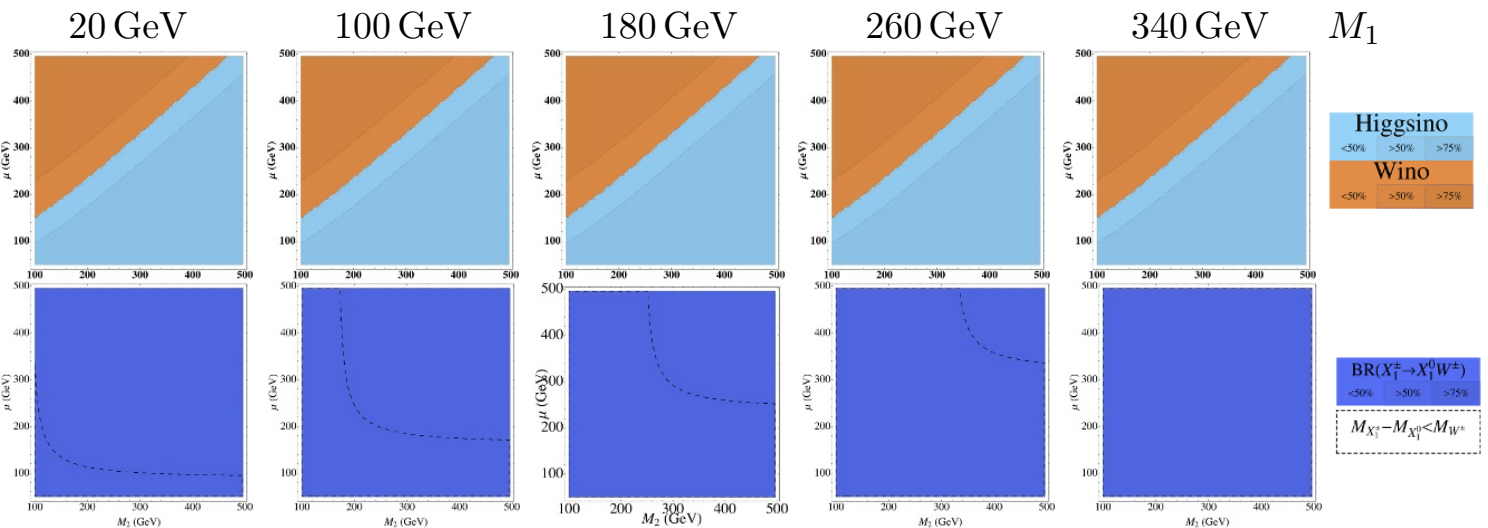

Figure 5. $\chi_{\mathbf{1}}^{ \pm}$: Dominant gauge eigenstate content (top) and leading decay modes (bottom) of the $\chi_{1}^{ \pm}$chargino in the $M_{2}-\mu$ plane for various slices of $M_{1}$ and $\tan \beta=10$. The thick, dashed lines indicate where the corresponding decay only occurs with an off-shell vector boson. Shaded, dash-enclosed regions indicate the boundary of $50 \%$ and $75 \%$ composition/branching ratio, as noted in the legend.

amplitude while $\widetilde{B}^{0} \rightarrow h^{0} \widetilde{W}^{0}$ requires only one. While this would seem to favour the Higgs mode when all three can occur on-shell, the gauge modes are found to be comparable or even more likely. This follows from the Goldstone boson equivalence theorem [69-71]. For large mass splittings $\Delta M$, the decay rates to massive vectors are enhanced by a factor on the order of $\left(\Delta M / m_{Z}\right)^{2}$ relative to the Higgs channel, and this effectively cancels the additional mixing factor appearing in the amplitudes for the gauge modes [72]. Note that this enhancement is present only when the intial and final states have a mass splitting parametrically larger than $m_{Z}$. In particular, no such enhancement occurs for vector boson decays involving Higgsino to Higgsino or Wino to Wino states.

The dominant gauge-eigenstate component of the $\chi_{1}^{0}$ neutralino is shown in figure 4 . Unsurprisingly, it nearly always corresponds to the smallest of the underlying neutralino mass parameters. When the $\chi_{1}^{0}$ is mostly Higgsino, it coincides with the $\widetilde{H}_{-}^{0}$ linear combination (for $\mu, M_{1}$, and $M_{2}$ of the same sign). This state is stable by assumption, and there are no decay modes to be shown. 

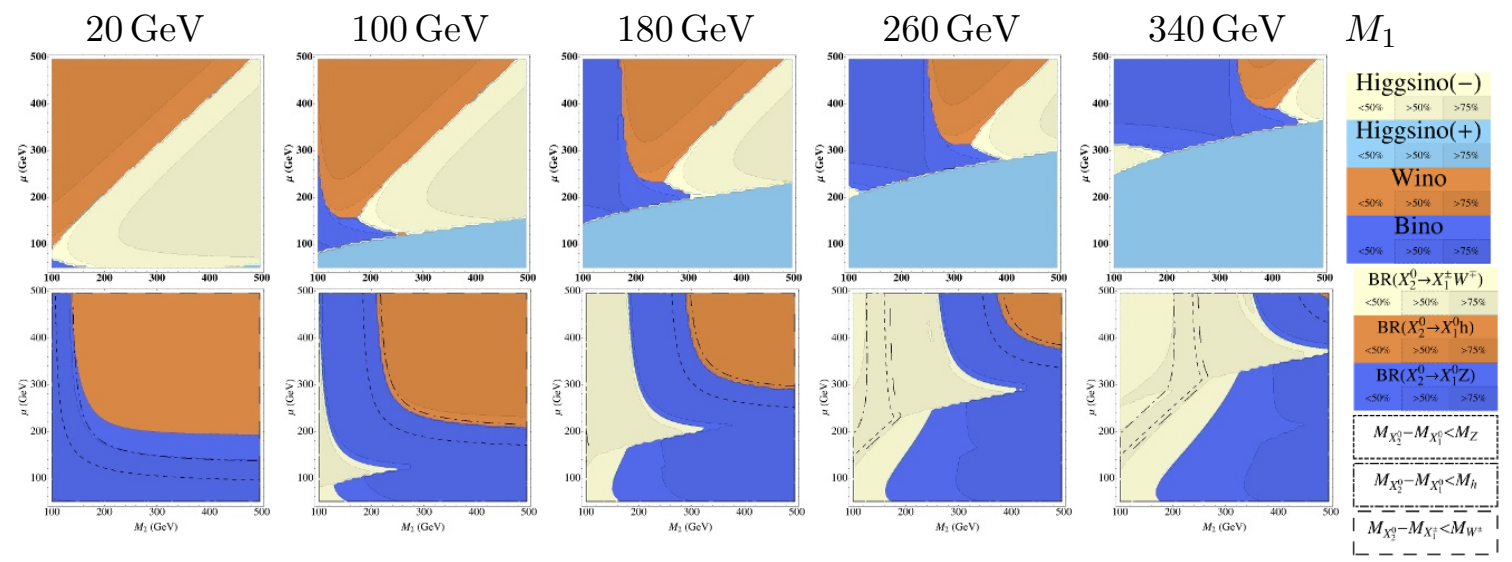

Figure 6. $\chi_{\mathbf{2}}^{\mathbf{0}}$ : Dominant gauge eigenstate content (top) and leading decay modes (bottom) of the $\chi_{2}^{0}$ neutralino in the $M_{2}-\mu$ plane for various slices of $M_{1}$ and $\tan \beta=10$. The thick, dashed and dotted lines indicate where the corresponding decays only occur with an off-shell vector boson. Shaded, dash-enclosed regions indicate the boundary of $50 \%$ and $75 \%$ composition/branching ratio, as noted in the legend.
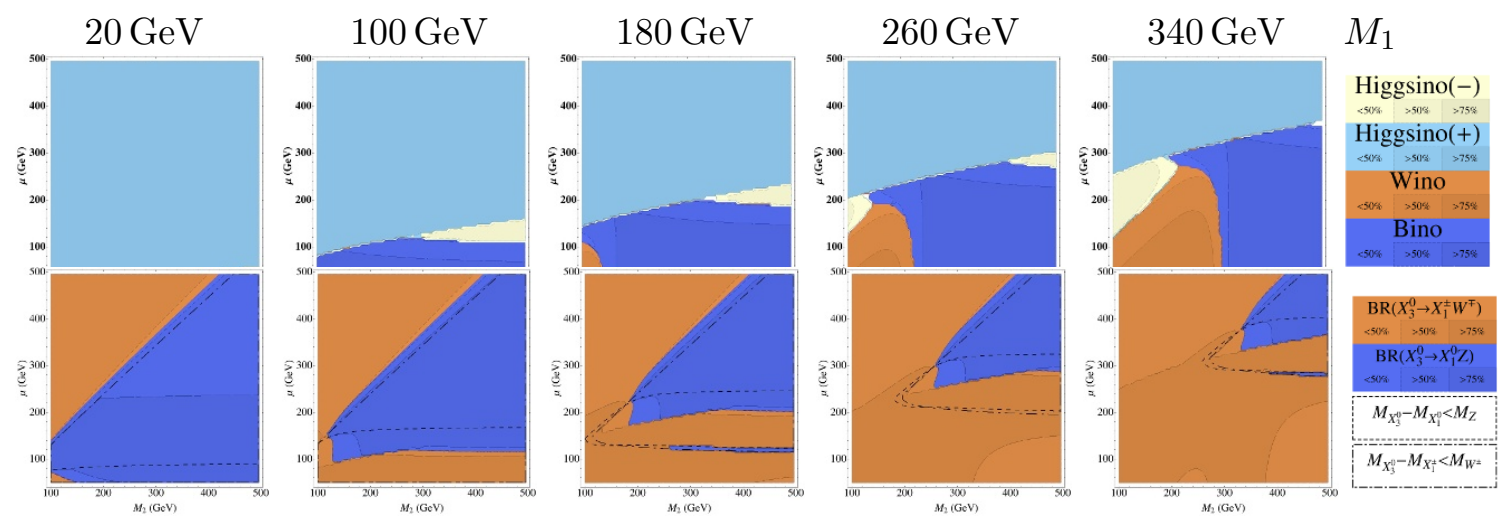

Figure 7. $\chi_{\mathbf{3}}^{\mathbf{0}}$ : Dominant gauge eigenstate content (top) and leading decay modes (bottom) of the $\chi_{3}^{0}$ neutralino in the $M_{2}-\mu$ plane for various slices of $M_{1}$ and $\tan \beta=10$. The thick, dashed and dotted lines indicate where the corresponding decays only occur with an off-shell vector boson. Shaded, dash-enclosed regions indicate the boundary of $50 \%$ and $75 \%$ composition/branching ratio, as noted in the legend.

In figure 5, we show the gauge content and the leading decay modes of the lighter chargino, $\chi_{1}^{ \pm}$. For our choice of positive signs for $\mu, M_{1}$, and $M_{2}$, we find that it is always the next-to-lightest state in the spectrum. For this reason, the only available decay mode is $\chi_{1}^{ \pm} \rightarrow W^{ \pm(*)} \chi_{1}^{0}$, as can be seen in the lower panels of the figure. The dashed lines in these plots show the boundary between where this decay occurs with the $W^{ \pm}$off or on shell. This line lies slightly above the contour in figure 4 where the lightest $\chi_{1}^{0}$ state is Bino-like. When this is not the case, the $\chi_{1}^{ \pm}$and $\chi_{1}^{0}$ modes are typically both Wino-like or Higgsino-like and the mass splitting between them is less than $m_{W}$. 

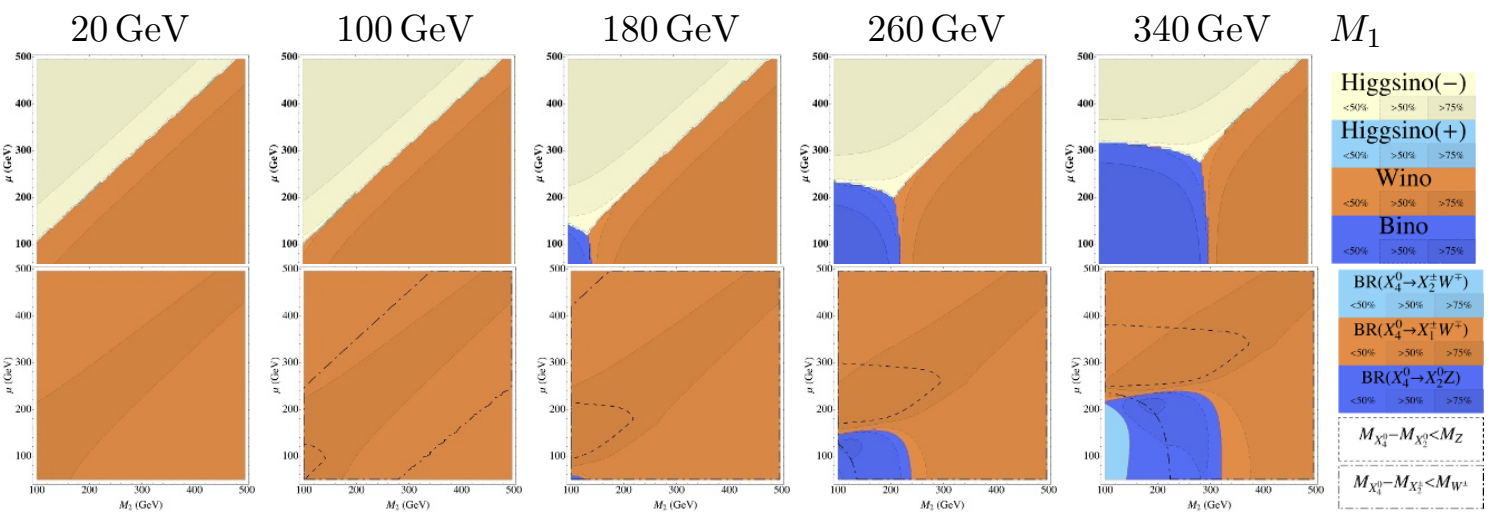

Figure 8. $\chi_{\mathbf{4}}^{\mathbf{0}}$ : Dominant gauge eigenstate content (top) and leading decay modes (bottom) of the $\chi_{4}^{0}$ neutralino in the $M_{2}-\mu$ plane for various slices of $M_{1}$ and $\tan \beta=10$. The thick, dashed and dotted lines indicate where the corresponding decays only occur with an off-shell vector boson. Shaded, dash-enclosed regions indicate the boundary of $50 \%$ and $75 \%$ composition/branching ratio, as noted in the legend.
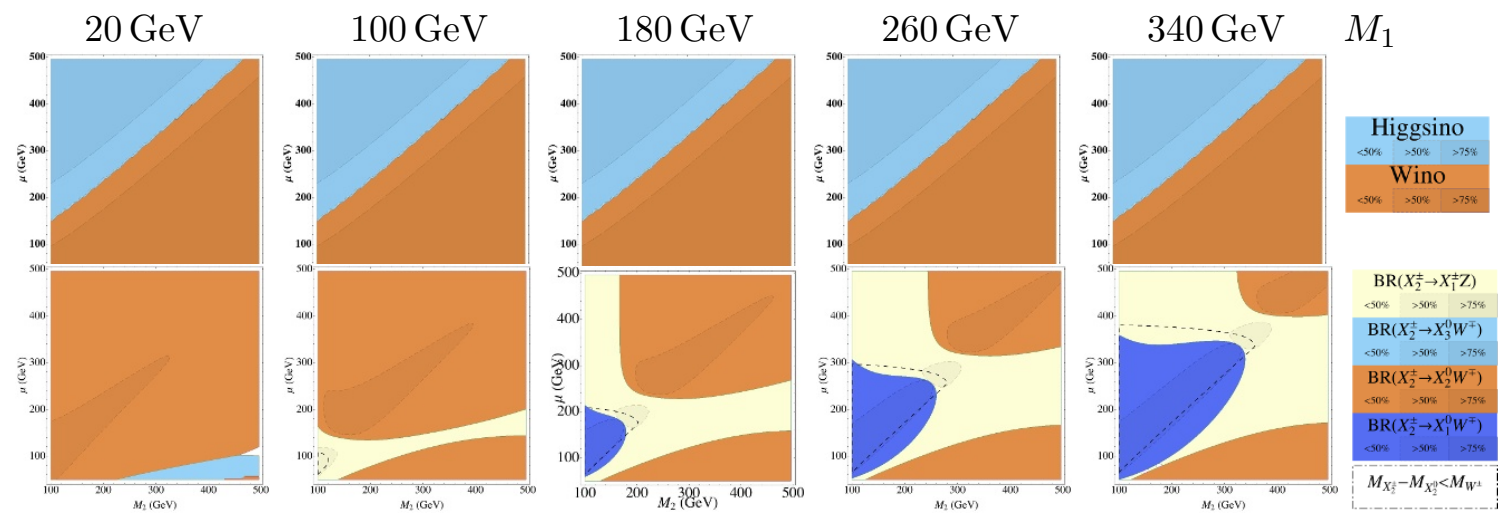

Figure 9. $\chi_{2}^{ \pm}$Dominant gauge eigenstate content (top) and leading decay modes (bottom) of the $\chi_{2}^{ \pm}$chargino in the $M_{2}-\mu$ plane for various slices of $M_{1}$ and $\tan \beta=10$. The thick, dashed and dotted lines indicate where the corresponding decays only occur with an off-shell vector boson. Shaded, dash-enclosed regions indicate the boundary of $50 \%$ and $75 \%$ composition/branching ratio, as noted in the legend.

The content and decays of the $\chi_{2}^{0}$ neutralino are shown in figure 6 . Three distinct decay modes are now possible, and the thick dashed and dotted lines in the lower panels illustrate where they can occur on-shell. The decay $\chi_{2}^{0} \rightarrow h^{0} \chi_{1}^{0}$ is seen to dominate in the upper right corner of these plots when $\chi_{2}^{0}$ is Wino- or Higgsino-like and $\chi_{1}^{0}$ is Bino-like. The related decay with a $Z^{0}$ typically has a similar (but smaller) branching in this region. It requires an additional mixing factor relative to the Higgs mode, but can also receive a Goldstone boson enhancement. On the other hand, no such enhancement occurs for $\chi_{2}^{0} \rightarrow W^{\mp} \chi_{1}^{ \pm}$in this region, since both states are close in mass, and the corresponding branching ratio is negligible. Note as well that the Higgs decay dominates only when it is two-body due to 
the very small width of the Higgs. Vector boson modes are dominant in the rest of the parameter space. When the $\chi_{2}^{0}$ state is Wino-like, it tends to be very degenerate with the $\chi_{1}^{ \pm}$, and so the $Z^{0} \chi_{1}^{0}$ channel dominates due to the larger available phase space. For a Bino- or Higgsino-like $\chi_{2}^{0}$ state, the neutral and charged vector modes tend to have similar branchings, with the Higgs mode contributing at a significant (but sub-leading) level when it can occur on-shell.

The leading components and decay channels of $\chi_{3}^{0}$ are shown in figure 7 . The $\chi_{3}^{0} \rightarrow$ $Z^{0} \chi_{1}^{0}$ mode dominates when it can occur on-shell but $\chi_{3}^{0} \rightarrow W^{\mp} \chi_{1}^{ \pm}$cannot. This occurs when $\left|M_{1}\right|<|\mu|<\left|M_{2}\right|$. Otherwise, the $W^{\mp}$ is dominant, although $\chi_{3}^{0} \rightarrow h^{0} \chi_{1}^{0}$ can be significant as well when it can occur on-shell.

In figure 8, we show the content and leading decay modes of the heaviest neutralino $\chi_{4}^{0}$. The dominant decay channel is to the kinematically unsuppressed $\chi_{4}^{0} \rightarrow \chi_{1}^{ \pm} W^{\mp}$ in the regions where the $\chi_{4}^{0}$ has a significant Higgsino or Wino components. For intermediate values of $M_{1}$, where the LSP is either Wino-like or Higgsino-like and either $\chi_{2}^{0}$ or $\chi_{3}^{0}$ is Bino-like, the production of $\chi_{4}^{0} \chi_{2}^{ \pm}$is important, as lighter modes will be either suppressed (Bino production) or result in soft decays with low acceptance rates. In the region where $\chi_{4}^{0}$ is significantly Bino-like, the branching ratio is split between all unsuppressed modes with one mixing $\left(\chi_{2}^{ \pm} W^{\mp}, \chi_{1}^{ \pm} W^{\mp}, \chi_{2}^{0} Z\right)$, with the largest component (though $<50 \%$ ) to the Wino-like $\chi_{2}^{ \pm}$. As the mass of the $\chi_{2}^{ \pm}$increases, the $\chi_{2}^{ \pm} W^{\mp}$ mode becomes kinematically suppressed, and the Higgsino-like $\chi_{2}^{0} Z^{0}$ mode dominates over the Higgsino-like $\chi_{1}^{ \pm} W^{\mp}$ for the remainder of the region with a Bino-like $\chi_{4}^{0}$.

Finally, we show the dominant components and leading decay modes of the heavier chargino $\chi_{2}^{ \pm}$in figure 9. Production of the $\chi_{2}^{ \pm}$is important where the LSP is either Higgsinolike or Wino-like, since the $\chi_{1}^{ \pm}$state will decay to soft leptons in this region, as indicated in figure 5. The decays of the $\chi_{2}^{ \pm}$are relatively uniformly split between $\chi_{1}^{ \pm} Z, \chi_{2}^{0} W^{ \pm}$and $\chi_{1}^{0} W^{ \pm}$, as very little of the parameter space shows branching ratios larger than $50 \%$.

We have also examined the dependence of these decay fractions on $\tan \beta$ in the range $2 \leq \tan \beta \leq 50$. The results throughout this wide range are qualitatively very similar to the $\tan \beta=10$ case that we have studied in detail.

\subsection{Implications for LHC signals}

Before turning to a detailed analysis of the sensitivity of LHC searches to electroweakinos, let us briefly emphasize three points that will be important in the analysis to follow. First, production rates tend to be greatest for the lightest pairs of states with significant Higgsino or Wino components, and the subsequent cascade decays are usually fairly short. This motivates searches for relatively simple decay topologies. Second, in a very significant fraction of the parameter space, the leading decay modes occur between states with mass splittings less than $m_{Z}$ or $m_{W}$. As a result, the decay products frequently have low $p_{T}$, and invariant mass pairings that do not reconstruct a resonance (or a kinematic edge). This limits the sensitivity of searches that attempt to reconstruct specific kinematic features characteristic of on-shell vector boson decays or large missing energy. And third, many states are found to have multiple relevant decay modes. This implies that the full 
inclusive signals of MSSM electroweakinos can be much richer and more complicated than the simplified-model realizations that are frequently applied (e.g. ref. [24]).

\section{$3 \quad$ Methodology of LHC sensitivity estimates}

We turn next to investigate the sensitivity of ATLAS and CMS searches to neutralinos and charginos. Both collaborations have explored a wide variety of possible SUSY signals, including specific searches geared towards the electroweakinos. In this section we describe the techniques we used to apply these and more general searches to the MSSM. Our results will be presented in the section to follow.

Signal events were generated independently for all 21 possible production pairings using MadGraph5 [63] interfaced with Pythia 6.4 [73]. Hard scattering processes with zero or one additional jets $\left(p p \rightarrow \chi_{i} \chi_{j}+\{0,1\} j\right)$ were obtained from MadGraph5 and passed to Pythia 6.4 to be decayed, showered, and hadronized, with the inclusion of MLM matching between additional hard jets and the parton shower [74]. For each MSSM parameter point and each inclusive production channel, 50000 events were generated. These events were then passed to the Delphes 3 detector simulator [75], with triggers, jet reconstruction (anti$\mathrm{kT}$ ), and hadronic/leptonic tagging efficiencies modified to match the specifications for each experimental search channel considered. The results from all 21 production channels were combined for each search to obtain the inclusive MSSM signal by weighting each channel by its net cross section after matching and cuts.

The cuts implemented in each search channel in each analysis were reproduced from the information provided by Delphes. All analyses were vetted against cut-flow tables where provided by the experimental groups. To account for pile-up, the $\mathbb{E}_{T}$ values extracted from Delphes were smeared in an additional post-processing step, which was found to be necessary in the vetting process. Specifically, a Gaussian smearing was applied to the Delphes $\mathbb{E}_{T}$ values with a standard deviation of 0.75 times the value given in ref. [76], where the multiplicative factor compensates for the smearing already present in Delphes. ${ }^{2}$ Values of the $m_{T 2}$ variable used in some of the analyses were computed using the MT2_Bisect package [78, 79], while the Razor variables of ref. [80] were calculated using the algorithm provided by the CMS collaboration.

Two superimposed grids of points were generated in the $M_{2}-\mu$ plane, with a $5 \times 5$ grid of $M_{2}(100-500 \mathrm{GeV})$ and $\mu(50-500 \mathrm{GeV})$, and a $4 \times 4$ grid of $M_{2}(140-440 \mathrm{GeV})$ and $\mu(95-433 \mathrm{GeV})$, for seven slices of $M_{1}(20,60,100,180,240,320,420 \mathrm{GeV})$. The $4 \times 4$ grid was critical in adding insight into the large regions between the rough $5 \times 5$ grid without significantly increasing the computation time, as would a more populated, uniform grid. The signals calculated at each grid point were then extended to form a uniform $9 \times 9$ grid using linear interpolation of the logarithm of the event rates, following which a threedimensional order-three polynomial interpolation was performed over the entire $7 \times 9 \times 9$ dataset, again on the logarithm of the event rates. Exclusion regions were then determined from comparison of the calculated number events to the $95 \%$ confidence level (C.L.) limit

\footnotetext{
${ }^{2}$ Ref. [77] found that modifying the Delphes $\mathbb{E}_{T}$ smearing by a post-processed Gaussian smearing with a standard deviation of $\sim 20 \mathrm{GeV}$ effectively reproduced the smearing effect at LHC 8 .
} 
on the number of signal events $\left(N_{i}^{95}\right)$. For the ATLAS studies, the $N_{i}^{95}$ were provided, while for the CMS studies, the $N_{i}^{95}$ were calculated using the $C L_{s}$ method [81] with Gaussiandistributed uncertainties as implemented in RooStats [82]. In our analysis, we combine the exclusion regions from each separate signal region in a boolean fashion.

From ATLAS, we investigated the following searches:

- opposite-sign dileptons with $\mathbb{E}_{T}$ and no jets [25]

- trilepton plus $\mathbb{E}_{T}[24,83]$;

- four or more leptons [84];

- dileptons with razor variables [85]

- hadronic di- $\tau$ plus $\mathbb{E}_{T}[86]$

- same-sign dileptons plus jets [87]

- monojet $[5,88]$.

- jets plus $\mathbb{E}_{T}[89]$;

- disappearing charged tracks [90]

From CMS, we considered the following studies:

- leptons (dilepton, trilepton, multi-lepton) with $\mathbb{E}_{T}[91]$

- chargino and neutralino search using $h \rightarrow \bar{b} b$ decays [23].

- monojet [92].

Many of the LHC searches use similar final states but different specific search strategies. In particular, ATLAS searches tend to focus on a small number of cut regions that are designed to enhance the signal from a specific simplified model, whereas CMS searches tend to be more broadly focused, arranging a grid of cut regions over a larger phase space. As a result, these searches are frequently complementary.

\section{Limits from the LHC}

Following the methods described above, we have derived exclusions on the parameter space of MSSM charginos and neutralinos using existing LHC searches. Many of the searches we apply were designed to find other superpartners (or forms of new physics), while others have only been used by the collaborations to constrain specific simplified models of the electroweakinos. We find that some of these searches also give new constraints on the more general MSSM electroweakino sector.

The most important LHC exclusions are shown in figures 10-13. These correspond to the ATLAS opposite-sign dilepton, trilepton, and four-plus lepton studies, together with the CMS lepton plus $b \bar{b}$ search, and will be discussed in more detail below. The thick black lines in the figures show the boundaries of the combined $95 \%$ confidence level (c.l.) exclusions in the $M_{2}-\mu$ plane for $\tan \beta=10$ and several values of $M_{1}$. The colour shading 
$M_{1}=20 \mathrm{GeV}$

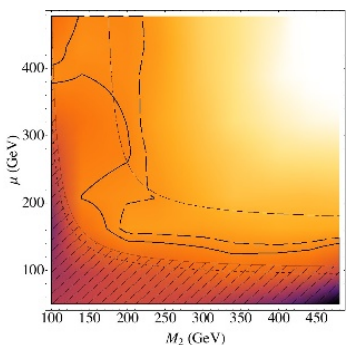

$60 \mathrm{GeV}$

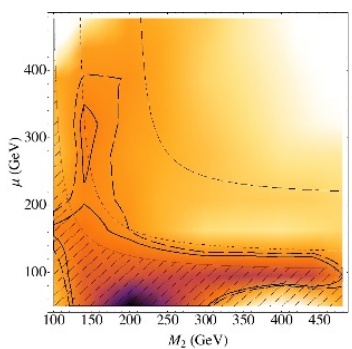

$100 \mathrm{GeV}$

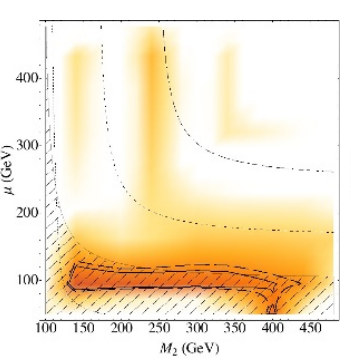

$180 \mathrm{GeV}$

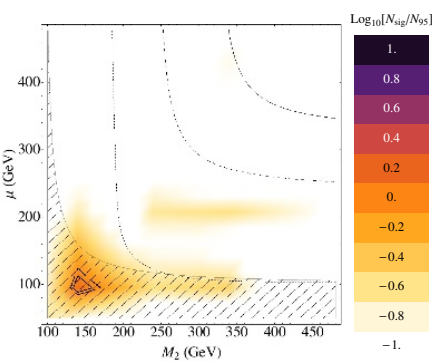

Figure 10. Parameter exclusions from the ATLAS opposite-sign dilepton search of ref. [25] in the $M_{2}-\mu$ plane for several fixed values of $M_{1}$. The boundaries of the $95 \%$ c.l. excluded regions are denoted by the thick black solid lines (thick black dashed lines) assuming a $K$ factor of 1.0 (1.2). Colour shading indicates the number of predicted signal events relative to the number excluded by the experimental analysis. The hatched area shows the $95 \%$ c.l. exclusion from LEP. Contours of constant $\Delta M=m_{\chi_{1}^{ \pm}}-m_{\chi_{1}^{0}}$ are indicated by the thin dashed lines - long dash: $\Delta M=2 m_{W}$; mid dash: $\Delta M=m_{W}$; short dash: $\Delta M=15 \mathrm{GeV}$.

indicates the number of predicted signal events (after cuts) relative to the number that are excluded by the corresponding experimental analyses. The hatched regions in figures $10-$ 13 indicate the $95 \%$ c.l. exclusions from the LEP experiments [93], which are close to $m_{\chi_{1}^{ \pm}}>103.4 \mathrm{GeV}$ for $\Delta M=m_{\chi_{1}^{ \pm}}-m_{\chi_{1}^{0}}>3 \mathrm{GeV}$ or $\Delta M<0.15 \mathrm{GeV}$ [93] but can fall to as low as $92.4 \mathrm{GeV}$ for mass differences between these boundaries [35, 94]. In these figures we also show contours of constant mass differences $\Delta M$ with thin dashed lines: long dash for $\Delta M=2 m_{x}$; mid dash for $\Delta M=m_{x}$; short dash for $\Delta M=15 \mathrm{GeV}-$ where $m_{x}=m_{W}$ and $\Delta M=m_{\chi_{1}^{ \pm}}-m_{\chi_{1}^{0}}$ for the lepton analyses in figure 10-11, $m_{x}=m_{Z}$ with $\Delta M=m_{\chi_{2}^{0}}-m_{\chi_{1}^{0}}$ in figure 12 , and $m_{x}=m_{h}$ with $\Delta M=m_{\chi_{2}^{0}}-m_{\chi_{1}^{0}}$ for the Higgsmotivated $b \bar{b}$ analysis in figure 13. These lines are useful for understanding constraints, as acceptance rates typically depend on whether decays occur on- or off-shell.

In setting these exclusions, we used the leading-order production cross sections obtained from MadGraph5. These were generally found to lie between the LO and NLO cross sections derived from Prospino2.1 [64, 65], and thus our exclusions are somewhat conservative. However, to illustrate the effects of slightly larger cross sections, we also show with thick solid dashed lines the boundaries of the regions excluded when a $K$-factor of 1.2 is applied to the MadGraph LO signal cross sections. This is typical of the ratio of NLO to LO cross sections computed with Prospino2.1.

\subsection{ATLAS opposite sign dileptons}

The ATLAS opposite-sign (OS) dilepton search of ref. [25] was designed to probe direct slepton and chargino production. Five distinct search regions were considered. All regions had a minimal requirement of two isolated OS leptons with $p_{T}>10 \mathrm{GeV}$ and $|\eta| \lesssim 2.4$, and no jets. Additional requirements were imposed on lepton $p_{T}, \mathbb{E}_{T}$, relative lepton flavour, and dileptonic kinematic variables. To suppress backgrounds, an effective $Z^{0}$ veto was imposed on all five regions, either by rejecting events with the leading dilepton invariant mass in the range $\left|m_{\ell \ell}-m_{Z}\right|<10 \mathrm{GeV}$, or by demanding that the leading OS leptons differ in flavour. In four of the five regions, a minimal requirement is imposed on the 

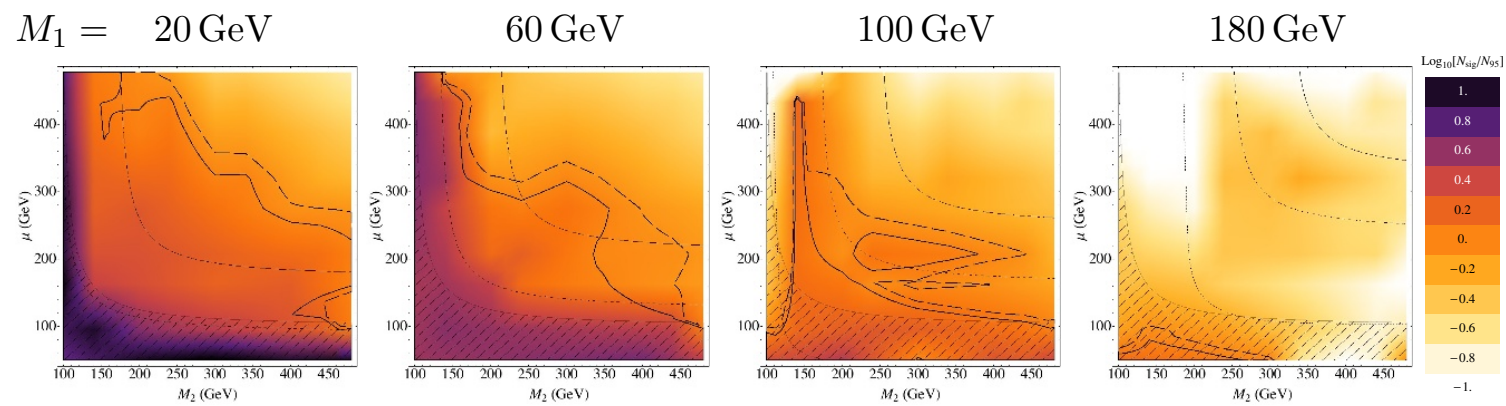

Figure 11. Parameter exclusions from the ATLAS trilepton search of ref. [24] in the $M_{2}-\mu$ plane for several fixed values of $M_{1}$. The boundaries of the $95 \%$ c.l. excluded regions are denoted by the thick black solid lines (thick black dashed lines) assuming a $K$ factor of 1.0 (1.2). Colour shading indicates the number of predicted signal events relative to the number excluded by the experimental analysis. The hatched area shows the $95 \%$ c.l. exclusion from LEP. Contours of constant $\Delta M=m_{\chi_{1}^{ \pm}}-m_{\chi_{1}^{0}}$ are indicated by the thin dashed lines - long dash: $\Delta M=2 m_{W}$; mid dash: $\Delta M=m_{W}$; short dash: $\Delta M=15 \mathrm{GeV}$.

variable $m_{T 2}>90 \mathrm{GeV}$, based on the dilepton system $[78,79] .{ }^{3}$ This is expected to have an endpoint at $m_{W}$ for SM backgrounds, while larger values can be obtained for chargino decays with $\left(m_{\chi_{1}^{ \pm}}-m_{\chi_{1}^{0}}\right) \gg m_{W}$. The fifth signal region does not impose a cut on $m_{T 2}$ but suffers from a much larger background rate.

The exclusions derived from this search for general electroweakino parameters are shown in figure 10. The strongest bounds are obtained for small values of $M_{1}$, and correspond mostly to the production of Wino- or Higgsino-like $\chi_{1}^{ \pm}$followed by decays to a Binolike LSP. Lower $M_{1}$ gives larger mass differences $\Delta M=m_{\chi_{1}^{ \pm}}-m_{\chi_{1}^{0}}$ for a given value of $\mu$ or $M_{2}$, which leads to more $\mathbb{E}_{T}$, larger $m_{T 2}$, and a higher fraction of electroweakino events passing the acceptance cuts. The larger production rate of Winos relative to Higgsinos (see figure 1) leads to a stronger exclusion when $M_{2}<\mu$. For $\mu \sim M_{2}$ and $M_{1}=60 \mathrm{GeV}$, the exclusions are increased slightly over the $\mu \gg M_{2}$ or $\mu \ll M_{2}$ regions due to contributions from $\chi_{1}^{0} \chi_{2}^{0}$ production where the decay chain $\chi_{2}^{0} \rightarrow \chi_{1}^{ \pm} W^{\mp} \rightarrow \chi_{1}^{0} W^{ \pm} W^{\mp}$ and off-shell $\chi_{2}^{0} \rightarrow \chi_{1}^{0} Z^{0}$ decays can also contribute to the signal regions.

Very little new exclusion beyond the LEP limit is found for $M_{1} \gtrsim 100 \mathrm{GeV}$. In this case, the LSP need not be Bino-like, and there is the possibility of dominantly Wino-toHiggsino or Higgsino-to-Wino transitions. However, the LEP chargino bounds force $\mu$ and $M_{2}$ to each be larger than about $100 \mathrm{GeV}$. Together with the reduced production rate at higher mass and the need for larger $\Delta M$ to pass the acceptance cuts, there is not enough data to probe this possibility using the dilepton analysis.

\subsection{ATLAS trilepton}

The ATLAS trilepton search [24] was designed in part to probe electroweakino production with decays through intermediate sleptons or weak vector bosons. Events with exactly three isolated leptons were selected. One pair must be same-flavour and opposite-sign (SFOS) with $m_{\ell \ell}>12 \mathrm{GeV}$, and events with $b$ jets were vetoed to suppress top backgrounds.

\footnotetext{
${ }^{3}$ In contrast to refs. [78, 79], it is computed here under the assumption of massless decay products.
} 

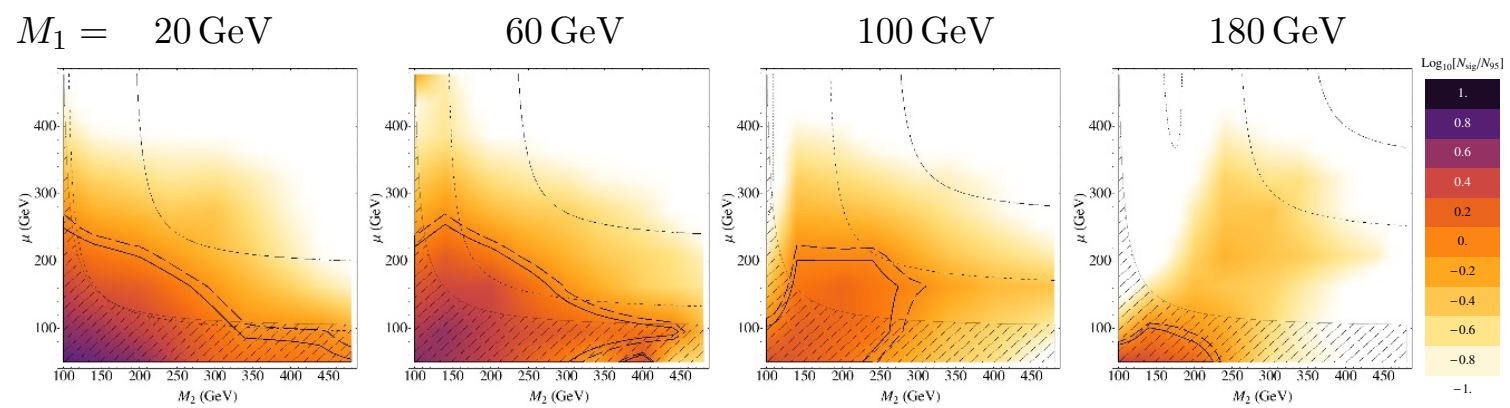

Figure 12. Parameter exclusions from the ATLAS four-plus lepton search of ref. [84] in the $M_{2}-\mu$ plane for several fixed values of $M_{1}$. The boundaries of the $95 \%$ c.l. excluded regions are denoted by the thick black solid lines (thick black dashed lines) assuming a $K$ factor of 1.0 (1.2). Colour shading indicates the number of predicted signal events relative to the number excluded by the experimental analysis. The hatched area shows the $95 \%$ c.l. exclusion from LEP. Contours of constant $\Delta M=m_{\chi_{2}^{0}}-m_{\chi_{1}^{0}}$ are indicated by the thin dashed lines - long dash: $\Delta M=2 m_{Z}$; mid dash: $\Delta M=m_{Z}$; short dash: $\Delta M=15 \mathrm{GeV}$.

Six exclusive search regions were defined with varying (but disjoint) requirements on the invariant mass of the SFOS pairing that is closest to $m_{Z}$, the $\mathbb{E}_{T}$, the $p_{T}$ of the lepton not included in the SFOS pairing, and the transverse mass $m_{T}$ of the unpaired lepton (for some signal regions).

The combined exclusions derived from this analysis are shown in figure 11. As in the OS dilepton search discussed above, the strongest limits are found for low $M_{1}$, where the dominant signal processes involve Wino- or Higgsino-like states decaying to a much lighter Bino-like LSP. Sensitivity is lost at larger $\mu$ or $M_{2}$ due to reduced production rates and the opening of decays involving Higgs bosons, which produce fewer leptons. Also as above, the sensitivity of this search is greatest for larger $\Delta M$. The interplay between production rates (smaller $M_{2}$ or $\mu$ ) and signal acceptance (larger $\Delta M$ ) can be seen in the $M_{1}=100 \mathrm{GeV}$ slice. In this slice, disjoint regions are excluded by separate signal regions that are sensitive to either on-shell $Z^{0}$ decays (isolated exclusion region) or off-shell $Z^{0}$ decays (bulk exclusion region). The gap between these regions is indicative of the reduced sensitivity of the study to the region where $m_{\chi_{2}^{0}}-m_{\chi_{1}^{0}} \sim m_{Z}$, which is also present in the results of [24].

For $M_{1}$ approaching $M_{2}$ and larger $\mu$, there is a rapid drop in the sensitivity of this search as seen in the upper portions of the $M_{1}=100,180 \mathrm{GeV}$ panels of figure 11 . In this region, the $\chi_{1}^{0}$ approaches the $\chi_{1}^{ \pm}$state in mass, leading to very soft leptons from $\chi_{1}^{ \pm} \rightarrow W^{+} \chi_{1}^{0}$ decays leading to a low acceptance in the trilepton search channels.

\subsection{ATLAS four lepton}

The ATLAS four-lepton search in ref. [84] was motivated by electroweakino production with decays through intermediate sleptons, R-parity violation, or to a gravitino and $Z^{0}$ boson. Four or more well-identified leptons were required, with up to one tau included in the count. Five search regions were defined, of which three have a $Z^{0}$ veto based on the invariant masses of SFOS pairs, with the other two regions demanding that a SFOS pair reconstruct a $Z^{0}$ to within $10 \mathrm{GeV}$. Additional requirements were imposed on $\mathbb{E}_{T}$ and $m_{\text {eff }}$ (defined to be the scalar sum of jet, lepton, and missing $p_{T}$ ). 

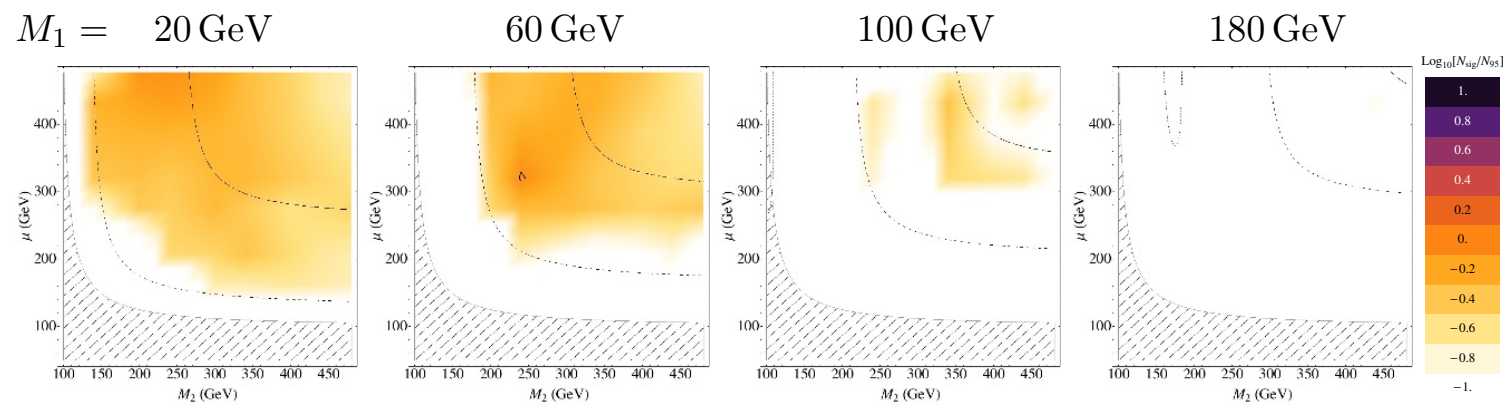

Figure 13. Parameter exclusions from the CMS lepton plus bottom quarks search of ref. [23] in the $M_{2}-\mu$ plane for several fixed values of $M_{1}$. The boundaries of the $95 \%$ c.l. excluded regions are denoted by the thick black solid lines (thick black dashed lines) assuming a $K$ factor of 1.0 (1.2). Colour shading indicates the number of predicted signal events relative to the number excluded by the experimental analysis. The hatched area shows the $95 \%$ c.l. exclusion from LEP. Contours of constant $\Delta M=m_{\chi_{2}^{0}}-m_{\chi_{1}^{0}}$ are indicated by the thin dashed lines - long dash: $\Delta M=2 m_{h}$; mid dash: $\Delta M=m_{h}$.

The exclusions derived from this search are illustrated in figure 12. The signal in this case can be generated by $\chi_{i}^{0} \chi_{j}^{0}$ production with both $\chi_{i, j}^{0} \rightarrow Z^{0(*)} \chi_{1}^{0}$ and $Z^{0(*)} \rightarrow \ell \bar{\ell}$, or through multistep cascades with $\chi_{i, j}^{0} \rightarrow W^{ \pm(*)} \chi_{1}^{\mp}$. We found that the most sensitive signal regions were SRnoZa and SRnoZb defined in ref. [84]. Recall that neutralino pair production relies on the Higgsino components of the states, and thus this study should be mainly sensitive to smaller values of $\mu$. In addition, $\mu \sim M_{2}$ results in a number of states with similar masses and significant Higgsino components, which increases the multiplicity of production modes that can contribute to the signal. As for the previous analyses, the sensitivity of this search falls off quickly with increasing $M_{1}$.

\subsection{CMS lepton plus bottom quarks}

The CMS lepton plus bottom quarks search of ref. [23] was designed to probe $\chi_{2}^{0} \chi_{1}^{ \pm}$production followed by $\chi_{2}^{0} \rightarrow h^{0} \chi_{1}^{0}$ with $h^{0} \rightarrow b \bar{b}$ and $\chi_{1}^{ \pm} \rightarrow W^{+(*)} \chi_{1}^{0}$ with $W^{+(*)} \rightarrow \ell \nu_{\ell}$. Events with one lepton, two b-tagged jets, and missing energy were selected. To suppress backgrounds from top quark production, a veto was imposed on additional leptons or jets along with a kinematic cut. Other backgrounds involving a leptonic $W$ were suppressed by demanding $m_{T}>100 \mathrm{GeV}$ for the lepton. The analysis also required a $b \bar{b}$ invariant mass in the range $100 \mathrm{GeV}<m_{b \bar{b}}<150 \mathrm{GeV}$ and applied a variable missing energy cut of $\mathbb{E}_{T}>100,125,150,175 \mathrm{GeV}$.

The sensitivity of this search to the general electroweakino parameter is shown in figure 13. In contrast to ref. [23], we do not find any excluded regions. The difference comes from our use of the computed $\chi_{2}^{0} \rightarrow h^{0} \chi_{1}^{0}$ branching ratio, whereas the CMS analysis assumes a branching fraction of one. As expected, a significant signal in this channel requires $\Delta M=m_{\chi_{2}^{0}}-m_{\chi_{1}^{0}}>m_{h}$, since off-shell decays involving the Higgs are very suppressed by its narrow width. Contours of $\Delta M=m_{h}\left(2 m_{h}\right)$ are indicated by mid (long) dashed lines in figure 13. For larger $M_{1}$ values, $\Delta M>m_{h}$ requires significantly heavy $\mu$ and $M_{2}$ and thus the sensitivity of currect LHC searches drops off quickly. 


\subsection{Other searches}

In addition to the four channels described above, we have investigated the sensitivity of a number of other LHC searches listed at the end of section 3. These give weaker exclusions, and we will only comment on them briefly.

The CMS collaboration has performed searches for two, three, and four leptons with missing energy in ref. [91] that are similar to the ATLAS studies considered above. In the CMS studies the signal region is subdivided into a large number of disjoint bins, whereas ATLAS uses a small number of signal regions geared towards specific decay cascades. Since we do not attempt to combine signal bins and only use boolean exclusions, the ATLAS limits are stronger. ATLAS has also performed a second trilepton analysis in ref. [83] with slightly different signal requirements than ref. [24] discussed above. We find similar bounds from ref. [83], and our trilepton-excluded region matches fairly well with their limit in the $M_{2}-\mu$ plane with low $M_{1}$.

We have also examined a broad range of searches that include one or more hard jets and missing energy among the selection requirements. These include the monojet $[5,88,92]$ and Razor analyses [85] that have been used to test dark matter production at the LHC [95-98], as well as channels with both hard jets and leptons [87]. The limits obtained from these are weaker than the lepton-centric studies above, with the typically high requirements on jet $p_{T}$ greatly reducing the electroweakino signal. In particular, we do not find any exclusion beyond the LEP limit from monojet searches, consistent with refs. [48, 49].

A qualitatively different analysis is the ATLAS search for disappearing charged tracks of ref. [90]. This search is sensitive to charginos that decay slowly to the lightest neutralino. Such long-lived charginos are expected to occur in the MSSM when $\left|M_{2}\right| \ll\left|M_{1}\right|$, $\mu$, as can occur in anomaly-mediated supersymmetry breaking [99, 100]. In this limit, the tree-level splitting between $\chi_{1}^{ \pm}$and $\chi_{1}^{0}$ is negligible, and the net mass splitting is dominated by loop effects that give $\Delta m \simeq 160 \mathrm{MeV}$ [101-103] This leads to a dominant $\chi_{1}^{ \pm} \rightarrow \pi^{-} \chi_{1}^{0}$ decay with a lifetime on the order of $0.1 \mathrm{~ns}$ [101]. For the moderate values of $\mu$ considered here, we find that the mass splitting between $\chi_{1}^{ \pm}$and $\chi_{1}^{0}$ is larger than $200 \mathrm{MeV}$, leading to lifetimes below the sensitivity of the ATLAS search. Larger values of $\mu$ than are explored in this study are needed to generate masses with a sufficiently compressed spectrum to be sensitive to bounds from ATLAS, and sufficiently large $M_{1}$ and $\mu$ can result in sensitivity up to $M_{2} \lesssim 260 \mathrm{GeV}$. We also find that the mass splitting can be smaller (or even negative [104]) when some of the mass parameters are negative.

\subsection{Combined exclusions}

Putting our results together, we show in figure 14 the combined sensitivity of all LHC searches considered in the $M_{2}-\mu$ plane for $M_{1}=20,60,100,180 \mathrm{GeV}$. The thick solid black line shows the $95 \%$ c.l. exclusion obtained using LO MadGraph production cross sections, while the dashed black line gives the exclusion when a signal $K$ factor of 1.2 is applied. The hatched region is excluded by LEP analyses. As expected, the excluded region is significant for small $M_{1}$, but shrinks quickly as $M_{1}$ is increased. 

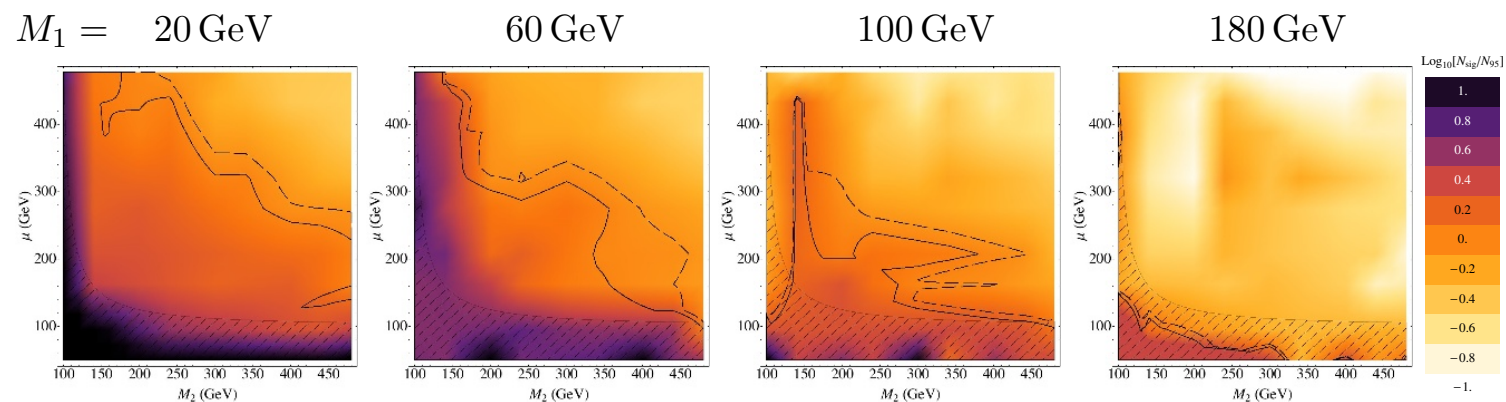

Figure 14. Combined exclusions from the LHC analyses discussed in the text in the $M_{2}-\mu$ plane for several fixed values of $M_{1}$. The boundaries of the $95 \%$ c.l. excluded regions are denoted by the thick black solid lines (thick black dashed lines) assuming a $K$ factor of 1.0 (1.2). Colour shading indicates the number of predicted signal events relative to the number excluded by the experimental analysis. The hatched area shows the $95 \%$ c.l. exclusion from LEP.

To investigate the $M_{1}$ dependence of these exclusions in more detail, we show in figure 15 the combined sensitivity of all LHC searches considered in the $M_{2}-M_{1}$ plane for $\mu=162,275,388,478 \mathrm{GeV}$. In each of these plots we also indicate the gaugino universality condition of $M_{2} \simeq 2 M_{1}$ with a blue dotted line. The excluded region only reaches to $M_{1} \sim 100 \mathrm{GeV}$. For larger $M_{1}$ values (and accounting for the LEP limits on charginos), either the mass splittings $\chi_{2}^{0}-\chi_{1}^{0}$ and $\chi_{1}^{ \pm}-\chi_{1}^{0}$ become small or the non-LSP states become heavy. Small mass splittings lead to a poor acceptance by the searches considered, while heavier non-LSP states are produced less frequently.

The excluded regions also shrink as $\mu$ becomes large. In particular, the exclusion in the $\mu=478 \mathrm{GeV}$ panel of figure 15, where the LSP is typically Bino-like and the $\chi_{1}^{ \pm}$and $\chi_{2}^{0}$ states are Wino-like, is much weaker than the exclusion than the exclusion quoted for a Bino-Wino simplified model in ref. [24]. In their analysis, they set $\operatorname{BR}\left(\chi_{2}^{0} \rightarrow Z^{0} \chi_{1}^{0}\right)=1$. In contrast, we find that in this limit the alternative decay mode $\chi_{2}^{0} \rightarrow h^{0} \chi_{1}^{0}$ can become very significant at large $\mu$. Since the Higgs boson $h^{0}$ decays only rarely produce more than a single lepton, this strongly suppresses the trilepton signal. ${ }^{4}$ Decreasing $\mu$ increases the probability of the $Z^{0}$ decay, and larger exclusions are found.

Note that in this work we have not examined the detailed dependence of the excluded regions on $\tan \beta$, having fixed its value to $\tan \beta=10$. However, as discussed previously, we find very similar production cross sections and decay branching fractions for $2<\tan \beta<50$. Thus, we expect qualitatively similar results for other values of $\tan \beta$.

\section{Conclusions}

In this work we have investigated the sensitivity of current LHC searches to the general chargino and neutralino sector of the MSSM in the limit where all the other superpartners are heavy enough to be neglected. This leaves a simple four-dimensional parameter space of $\left\{M_{1}, M_{2}, \mu, \tan \beta\right\}$. We have reinterpreted a diverse set of studies by ATLAS and CMS to derive exclusions on this space.

\footnotetext{
${ }^{4}$ We have also checked that our analysis methods give exclusions similar to ref. [24] when the Higgs decay mode is turned off.
} 
$\mu=162 \mathrm{GeV}$

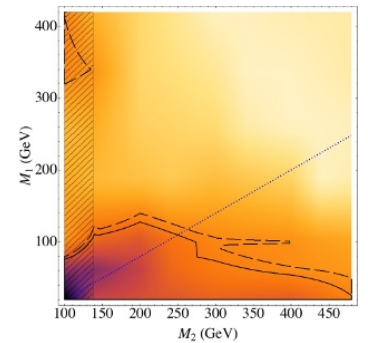

$275 \mathrm{GeV}$

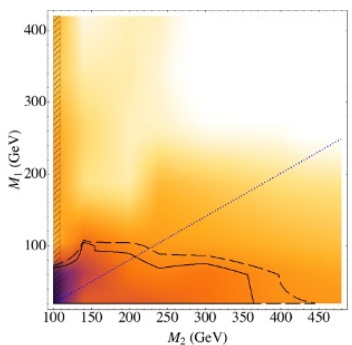

$388 \mathrm{GeV}$

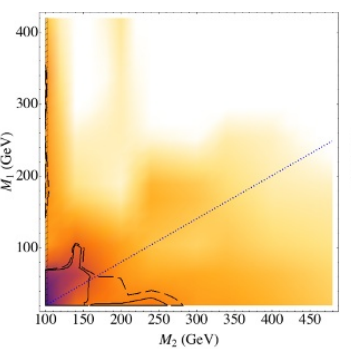

$478 \mathrm{GeV}$

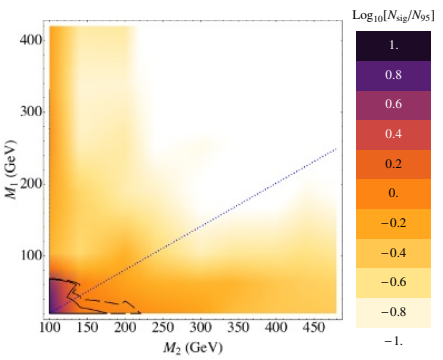

Figure 15. Combined exclusions from the LHC analyses discussed in the text in the $M_{2}-M_{1}$ plane for several fixed values of $\mu$. The boundaries of the $95 \%$ c.l. excluded regions are denoted by thick black solid lines (thick black dashed lines) assuming a $K$ factor of 1.0 (1.2). Colour shading indicates the number of predicted signal events relative to the number excluded by the experimental analysis. The hatched area shows the $95 \%$ c.l. exclusion from LEP. The dotted blue line indicates where $M_{2}=2 M_{1}$.

The greatest LHC sensitivity to general electroweakinos comes from searches requiring multiple leptons and missing energy. This helps to reduce the dominant background to vector diboson production. However, distinguishing the signal from electroweakinos from this remaining background is challenging, especially when the mass spectrum is compressed. For this reason, we only find significant parameter exclusions for relatively small values of $M_{1} \lesssim 100 \mathrm{GeV}$ with a Bino-like LSP. In this case, signals come from the production of heavier Wino- or Higgsino-like charginos and neutralinos followed by their decays to the LSP, primarily through weak vector bosons.

Despite the limited reach of existing LHC searches, our results show that they have a reasonable acceptance for larger electroweak masses. For this reason, we expect that much larger exclusions will be possible using similar analysis techniques with improved data sets from upcoming LHC runs. Additional data should also allow for the investigation of scenarios with a Wino- or Higgsino-like LSP. Further improvements may also be possible with modified analysis techniques, such as those proposed in refs. [44, 47, 105, 106].

Our results can be applied to test scenarios where the charginos and neutralinos play an important role. One example is dark matter, where the relic density is very sensitive to the gauge content of the LSP [38-40]. A second case is supersymmetric electroweak baryogenesis, in which the charginos and neutralinos are frequently the dominant new source of CP violation required for the net creation of baryons [107, 108]. In particular, our results suggest that the Bino-driven scenario of refs. [36, 37, 109] is not significantly constrained by current LHC data.

\section{Acknowledgments}

The authors would like to thank Anadi Canepa, Zoltan Gesce, Alejandro de la Puente, Isabel Trigger, and Peter Winslow for helpful discussions. This work was supported by the National Science and Engineering Research Council of Canada (NSERC) and by the Perimeter Institute for Theoretical Physics. Research at Perimeter Institute is supported 
by the Government of Canada through Industry Canada and by the Province of Ontario through the Ministry of Economic Development \& Innovation.

\section{A Mass matrices and mixings}

The charginos are mixtures of the charged components of the Winos and Higgsinos. Writing $\psi^{+}=\left(-i \widetilde{W}^{+}, \widetilde{H}_{u}^{+}\right)^{t}$ and $\psi^{-}=\left(-i \widetilde{W}^{-}, \widetilde{H}_{d}^{-}\right)^{t}$ the corresponding mass term (in twocomponent notation) is $[110,111]$

$$
-\mathscr{L}_{ \pm} \supset\left(\psi^{-}\right)^{t} X \psi^{+}+(\text {h.c. })
$$

with

$$
X=\left(\begin{array}{cc}
M_{2} & \sqrt{2} s_{\beta} m_{W} \\
\sqrt{2} c_{\beta} m_{W} & \mu
\end{array}\right) .
$$

The matrix $X$ is not Hermitian in general, so there may not exist a unitary matrix that diagonalizes it. However, it can always be bi-diagonalized with a pair of unitary matrices $U$ and $V$ such that

$$
V X^{\dagger} X V^{\dagger}=U^{*} X X^{\dagger} U^{t}=\operatorname{diag}\left(m_{\chi_{1}^{ \pm}}, m_{\chi_{2}^{ \pm}}\right),
$$

where $\left|m_{\chi_{1}^{ \pm}}\right| \leq\left|m_{\chi_{2}^{ \pm}}\right|$. In terms of $U$ and $V$, the mass and gauge eigenstates are related by

$$
\chi_{i}^{+}=V_{i j} \psi_{j}^{+}, \quad \chi_{i}^{-}=U_{i j} \psi_{j}^{-} .
$$

It is conventional to combine these into four-component Dirac fermions with $\chi_{i}^{+}=$ $\left(\chi_{i}^{+},\left(\chi_{i}^{-}\right)^{\dagger}\right)$ (in an obvious abuse of notation).

For the neutralinos, the mass term in the basis $\psi^{0}=\left(-i \widetilde{B}^{0},-i \widetilde{W}^{0}, \widetilde{H}_{d}, \widetilde{H}_{u}\right)^{t}$ is $[110$, $111]$

$$
-\mathscr{L} \supset \frac{1}{2}\left(\psi^{0}\right)^{t} Y \psi^{0}+(\text { h.c. })
$$

with

$$
Y=\left(\begin{array}{cccc}
M_{1} & 0 & -c_{\beta} s_{W} m_{Z} & s_{\beta} s_{W} m_{Z} \\
0 & M_{2} & c_{\beta} c_{W} m_{Z} & -s_{\beta} c_{W} m_{Z} \\
-c_{\beta} s_{W} m_{Z} & c_{\beta} c_{W} m_{Z} & 0 & -\mu \\
s_{\beta} s_{W} m_{Z} & -s_{\beta} c_{W} m_{Z} & -\mu & 0
\end{array}\right) .
$$

This matrix is complex symmetric, and can be diagonalized by a unitary matrix $N$ such that

$$
N^{*} Y N^{\dagger}=\operatorname{diag}\left(m_{\chi_{1}^{0}}, m_{\chi_{2}^{0}}, m_{\chi_{3}^{0}}, m_{\chi_{4}^{0}}\right),
$$

where $\left|m_{\chi_{1}^{0}}\right| \leq\left|m_{\chi_{2}^{0}}\right| \leq\left|m_{\chi_{3}^{0}}\right| \leq\left|m_{\chi_{4}^{0}}\right|$. The mass eigenstates are related to the gauge eigenstates via

$$
\chi_{i}^{0}=N_{i j} \psi_{j}^{0} .
$$

These two-component fermions can be combined into four-component Majorana spinors $\chi_{i}^{0}=\left(\chi_{i}^{0},\left(\chi_{i}^{0}\right)^{\dagger}\right)^{t}$ (with another abuse of notation). 


\section{B Couplings to the standard model}

For our purposes, we need the couplings of the charginos and neutralinos to the weak vector bosons and the light SM-like Higgs boson.

\section{B.1 Vector boson couplings}

These can be found in refs. [110, 112]. We will write everything in four-component notation. $W^{-} \chi_{i}^{0} \chi_{j}^{+}:$

$$
-\mathscr{L} \supset-g W_{\mu}^{-} \bar{\chi}_{i}^{0} \gamma^{\mu}\left(\mathcal{O}_{i j}^{L} P_{L}+\mathcal{O}_{i j}^{R} P_{R}\right) \chi_{j}^{+}+(\text {h.c. }),
$$

where

$$
\begin{aligned}
& \mathcal{O}_{i j}^{L}=-\frac{1}{\sqrt{2}} N_{i 4} V_{j 2}^{*}+N_{i 2} V_{j 1}^{*}, \\
& \mathcal{O}_{i j}^{R}=\frac{1}{\sqrt{2}} N_{i 3}^{*} U_{j 2}+N_{i 2}^{*} U_{j 1},
\end{aligned}
$$

with $g$ the $\mathrm{SU}(2)_{L}$ gauge coupling. These terms derive from the $\mathrm{SU}(2)_{L}$-covariant derivatives of the Higgsinos (first terms) and the Wino (second terms).

$Z^{0} \chi_{i}^{-} \chi_{j}^{+}:$

$$
-\mathscr{L} \supset-\bar{g} Z_{\mu}^{0} \bar{\chi}_{i}^{+} \gamma^{\mu}\left(\mathcal{O}^{\prime}{ }_{i j}^{L} P_{L}+\mathcal{O}^{\prime}{ }_{i j}^{R} P_{R}\right) \chi_{j}^{+}
$$

where

$$
\begin{aligned}
\mathcal{O}^{\prime}{ }_{i j}^{L} & =-V_{i 1} V_{j 1}^{*}-\frac{1}{2} V_{i 2} V_{j 2}^{*}+\delta_{i j} s_{W}^{2}, \\
\mathcal{O}^{\prime}{ }_{i j}^{R} & =-U_{i 1}^{*} U_{j 1}-\frac{1}{2} U_{i 2}^{*} U_{j 2}+\delta_{i j} s_{W}^{2},
\end{aligned}
$$

with $\bar{g}=g / c_{W}$. These come from the $\mathrm{SU}(2)_{L} \times \mathrm{U}(1)_{Y}$ gauge couplings of the Winos (first terms) and Higgsinos (second terms).

$Z^{0} \chi_{i}^{0} \chi_{j}^{0}$ :

$$
-\mathscr{L} \supset-\frac{1}{2} \bar{g} Z_{\mu}^{0} \bar{\chi}_{i}^{0} \gamma^{\mu}\left(\mathcal{O}^{\prime \prime}{ }_{i j}^{L} P_{L}+\mathcal{O}^{\prime \prime}{ }_{i j}^{R} P_{R}\right) \chi_{j}^{0},
$$

where

$$
\begin{aligned}
\mathcal{O}_{i j}^{L} & =-\frac{1}{2} N_{i 3} N_{j 3}^{*}+\frac{1}{2} N_{i 4} N_{j 4}^{*}, \\
\mathcal{O}_{i j}^{R} & =-\left(\mathcal{O}^{\prime \prime}{ }_{i j}^{L}\right)^{*},
\end{aligned}
$$

with $\bar{g}=g / c_{W}$. Note that these couplings come only from the Higgsinos. The Bino has no gauge couplings at all, while the $\widetilde{W}^{0}$ has $t^{3}=0=Y$ and therefore does not couple to the $Z^{0}$. For $i=j$, this coupling is purely axial. It also vanishes for $i=j$ in the limit that $i$ corresponds to a pure Higgsino state. 
$\gamma \chi_{i}^{-} \chi_{j}^{+}$

$$
-\mathscr{L} \supset \text { e } A^{\mu} \bar{\chi}_{i}^{+} \gamma^{\mu} \chi_{i}^{+}
$$

which is purely diagonal and present only for the charginos due to conservation of electric charge. Off-diagonal couplings and couplings to neutralinos can only occur by way of higher-dimensional operators such as the electric and magnetic moment forms.

\section{B.2 (SM-like) Higgs couplings}

These are listed in refs. [113, 114]. We will focus exclusively on the couplings to the SM-like Higgs $h^{0}$. The corresponding mixing angles with the $H_{u}^{0}$ and $H_{d}^{0}$ gauge eigenstates are

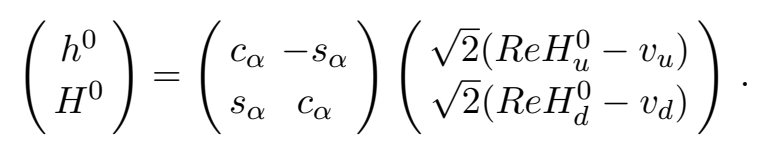

In the decoupling limit, the couplings of the lighter $h^{0}$ state to matter are identical to the SM. In this limit, the mixing angle reduces to $\alpha=\beta-\pi / 2$ so that $c_{\alpha}=s_{\beta}$ and $s_{\alpha}=-c_{\beta}$. $h^{0} \chi_{i}^{-} \chi_{j}^{+}:$

$$
-\mathscr{L} \supset g h^{0} \bar{\chi}_{i}^{+}\left[\left(c_{\beta} Q_{j i}^{*}+s_{\beta} S_{j i}^{*}\right) P_{L}+\left(c_{\beta} Q_{i j}+s_{\beta} S_{i j}\right) P_{R}\right] \chi_{j}^{+}
$$

with

$$
\begin{aligned}
Q_{i j} & =\frac{1}{\sqrt{2}} V_{i 1} U_{j 2}, \\
S_{i j} & =\frac{1}{\sqrt{2}} V_{i 2} U_{j 1} .
\end{aligned}
$$

These couplings involve one Higgsino component and one Wino component. They come from the $-i \sqrt{2} g \widetilde{W}^{a} H_{a}^{*} t^{a} \widetilde{H}_{a}$ supersymmetrizations of the Higgs boson gauge couplings.

$h^{0} \chi_{i}^{0} \chi_{j}^{0}:$

$$
-\mathscr{L} \supset g h^{0} \bar{\chi}_{i}^{0}\left[\left(c_{\beta} Q_{j i}^{\prime \prime *}-s_{\beta} S_{j i}^{\prime \prime}{ }_{j i}\right) P_{L}+\left(c_{\beta} Q^{\prime \prime}{ }_{i j}-s_{\beta} S^{\prime \prime}{ }_{i j}\right) P_{R}\right] \chi_{j}^{0}
$$

with

$$
\begin{aligned}
Q^{\prime \prime}{ }_{i j} & =\frac{1}{2}\left[N_{i 3}\left(N_{j 2}-t_{W} N_{j 1}\right)+N_{j 3}\left(N_{i 2}-t_{W} N_{i 1}\right)\right] \epsilon_{i}, \\
S^{\prime \prime}{ }_{i j} & =\frac{1}{2}\left[N_{i 4}\left(N_{j 2}-t_{W} N_{j 1}\right)+N_{j 4}\left(N_{i 2}-t_{W} N_{i 1}\right)\right] \epsilon_{i},
\end{aligned}
$$

where $\epsilon_{i}$ is the sign of the $i$-th mass eigenvalues (for real parameters). As above, these couplings involve one Higgsino component and one Wino or Bino component, and they come from the supersymmetrizations of the Higgs boson gauge couplings.

Open Access. This article is distributed under the terms of the Creative Commons Attribution License (CC-BY 4.0), which permits any use, distribution and reproduction in any medium, provided the original author(s) and source are credited. 


\section{References}

[1] CMS collaboration, Search for new physics in the multijet and missing transverse momentum final state in proton-proton collisions at $\sqrt{s}=8 \mathrm{Te}$, JHEP 06 (2014) 055 [arXiv:1402.4770] [INSPIRE].

[2] ATLAS collaboration, Search for squarks and gluinos with the ATLAS detector in final states with jets and missing transverse momentum using $\sqrt{s}=8$ TeV proton-proton collision data, JHEP 09 (2014) 176 [arXiv:1405.7875] [INSPIRE].

[3] R. Barbieri and G.F. Giudice, Upper bounds on supersymmetric particle masses, Nucl. Phys. B 306 (1988) 63 [INSPIRE].

[4] ATLAS collaboration, Search for direct third-generation squark pair production in final states with missing transverse momentum and two b-jets in $\sqrt{s}=8$ TeV pp collisions with the ATLAS detector, JHEP 10 (2013) 189 [arXiv:1308.2631] [INSPIRE].

[5] ATLAS collaboration, Search for pair-produced top squarks decaying into a charm quark and the lightest neutralinos with $20.3 \mathrm{fb}^{-1}$ of pp collisions at $\sqrt{s}=8$ TeV with the ATLAS detector at the LHC, ATLAS-CONF-2013-068, CERN, Geneva Switzerland (2013).

[6] ATLAS collaboration, Search for direct top-squark pair production in final states with two leptons in pp collisions at $\sqrt{s}=8$ TeV with the ATLAS detector, JHEP 06 (2014) 124 [arXiv:1403.4853] [INSPIRE].

[7] CMS collaboration, Search for top-squark pair production in the single-lepton final state in pp collisions at $\sqrt{s}=8 \mathrm{TeV}$, Eur. Phys. J. C 73 (2013) 2677 [arXiv:1308.1586] [INSPIRE].

[8] CMS collaboration, Search for supersymmetry using razor variables in events with b-jets in pp collisions at 8 TeV, CMS-PAS-SUS-13-004, CERN, Geneva Switzerland (2013).

[9] L.J. Hall and L. Randall, Weak scale effective supersymmetry, Phys. Rev. Lett. 65 (1990) 2939 [INSPIRE].

[10] R. Essig, E. Izaguirre, J. Kaplan and J.G. Wacker, Heavy flavor simplified models at the LHC, JHEP 01 (2012) 074 [arXiv: 1110.6443] [INSPIRE].

[11] C. Brust, A. Katz, S. Lawrence and R. Sundrum, SUSY, the third generation and the LHC, JHEP 03 (2012) 103 [arXiv:1110.6670] [InSPIRE].

[12] M. Papucci, J.T. Ruderman and A. Weiler, Natural SUSY endures, JHEP 09 (2012) 035 [arXiv:1110.6926] [INSPIRE].

[13] H. Baer et al., Radiative natural supersymmetry: reconciling electroweak fine-tuning and the Higgs boson mass, Phys. Rev. D 87 (2013) 115028 [arXiv:1212.2655] [INSPIRE].

[14] J.D. Wells, PeV-scale supersymmetry, Phys. Rev. D 71 (2005) 015013 [hep-ph/0411041] [INSPIRE].

[15] N. Arkani-Hamed and S. Dimopoulos, Supersymmetric unification without low energy supersymmetry and signatures for fine-tuning at the LHC, JHEP 06 (2005) 073 [hep-th/0405159] [INSPIRE].

[16] G.F. Giudice and A. Romanino, Split supersymmetry, Nucl. Phys. B 699 (2004) 65 [Erratum ibid. B 706 (2005) 65] [hep-ph/0406088] [INSPIRE].

[17] N. Arkani-Hamed, S. Dimopoulos, G.F. Giudice and A. Romanino, Aspects of split supersymmetry, Nucl. Phys. B 709 (2005) 3 [hep-ph/0409232] [INSPIRE]. 
[18] L.J. Hall and Y. Nomura, Spread supersymmetry, JHEP 01 (2012) 082 [arXiv:1111.4519] [INSPIRE].

[19] M. Ibe and T.T. Yanagida, The lightest Higgs boson mass in pure gravity mediation model, Phys. Lett. B 709 (2012) 374 [arXiv:1112.2462] [InSPIRE].

[20] M. Ibe, S. Matsumoto and T.T. Yanagida, Pure gravity mediation with $m_{3 / 2}=10-100 \mathrm{TeV}$, Phys. Rev. D 85 (2012) 095011 [arXiv: 1202.2253] [INSPIRE].

[21] A. Arvanitaki, N. Craig, S. Dimopoulos and G. Villadoro, Mini-split, JHEP 02 (2013) 126 [arXiv:1210.0555] [INSPIRE].

[22] N. Arkani-Hamed, A. Gupta, D.E. Kaplan, N. Weiner and T. Zorawski, Simply unnatural supersymmetry, arXiv:1212.6971 [INSPIRE].

[23] CMS collaboration, Search for electroweak production of charginos and neutralinos in final states with a Higgs boson in pp collisions at $8 \mathrm{TeV}$, CMS-PAS-SUS-13-017, CERN, Geneva Switzerland (2013).

[24] ATLAS collaboration, Search for direct production of charginos and neutralinos in events with three leptons and missing transverse momentum in $21 \mathrm{fb}^{-1}$ of pp collisions at $\sqrt{s}=8 \mathrm{TeV}$ with the ATLAS detector, ATLAS-CONF-2013-035, CERN, Geneva Switzerland (2013).

[25] ATLAS collaboration, Search for direct-slepton and direct-chargino production in final states with two opposite-sign leptons, missing transverse momentum and no jets in $20 \mathrm{fb}^{-1}$ of pp collisions at $\sqrt{s}=8 \mathrm{TeV}$ with the ATLAS detector, ATLAS-CONF-2013-049, CERN, Geneva Switzerland (2013).

[26] J. Alwall, P. Schuster and N. Toro, Simplified models for a first characterization of new physics at the LHC, Phys. Rev. D 79 (2009) 075020 [arXiv:0810.3921] [INSPIRE].

[27] LhC New Physics Working Group collaboration, D. Alves et al., Simplified models for LHC new physics searches, J. Phys. G 39 (2012) 105005 [arXiv:1105. 2838] [INSPIRE].

[28] M. Drees, H. Dreiner, D. Schmeier, J. Tattersall and J.S. Kim, CheckMATE: confronting your favourite new physics model with LHC data, arXiv:1312.2591 [INSPIRE].

[29] S. Kraml et al., SModelS: a tool for interpreting simplified-model results from the LHC and its application to supersymmetry, Eur. Phys. J. C 74 (2014) 2868 [arXiv:1312.4175] [INSPIRE].

[30] M. Papucci, K. Sakurai, A. Weiler and L. Zeune, Fastlim: a fast LHC limit calculator, Eur. Phys. J. C 74 (2014) 3163 [arXiv:1402.0492] [InSPIRE].

[31] J. Barnard and B. Farmer, A simple technique for combining simplified models and its application to direct stop production, JHEP 06 (2014) 132 [arXiv: 1402.3298] [INSPIRE].

[32] A. Bharucha, S. Heinemeyer and F. von der Pahlen, Direct chargino-neutralino production at the LHC: interpreting the exclusion limits in the complex MSSM, Eur. Phys. J. C 73 (2013) 2629 [arXiv: 1307.4237] [INSPIRE].

[33] S.P. Martin, K. Tobe and J.D. Wells, Virtual effects of light gauginos and higgsinos: a precision electroweak analysis of split supersymmetry, Phys. Rev. D 71 (2005) 073014 [hep-ph/0412424] [INSPIRE]. 
[34] J.A. Casas, J.M. Moreno, K. Rolbiecki and B. Zaldivar, Implications of light charginos for Higgs observables, LHC searches and dark matter, JHEP 09 (2013) 099 [arXiv:1305.3274] [INSPIRE].

[35] B. Batell, S. Jung and C.E.M. Wagner, Very light charginos and Higgs decays, JHEP 12 (2013) 075 [arXiv: 1309.2297] [InSPIRE].

[36] V. Cirigliano, Y. Li, S. Profumo and M.J. Ramsey-Musolf, MSSM baryogenesis and electric dipole moments: an update on the phenomenology, JHEP 01 (2010) 002 [arXiv:0910.4589] [INSPIRE].

[37] J. Kozaczuk and S. Profumo, Closing in on supersymmetric electroweak baryogenesis with dark matter searches and the Large Hadron Collider, JCAP 11 (2011) 031 [arXiv: 1108.0393] [INSPIRE].

[38] N. Arkani-Hamed, A. Delgado and G.F. Giudice, The well-tempered neutralino, Nucl. Phys. B 741 (2006) 108 [hep-ph/0601041] [InSPIRE].

[39] G.F. Giudice, T. Han, K. Wang and L.-T. Wang, Nearly degenerate gauginos and dark matter at the LHC, Phys. Rev. D 81 (2010) 115011 [arXiv: 1004.4902] [INSPIRE].

[40] C. Cheung, L.J. Hall, D. Pinner and J.T. Ruderman, Prospects and blind spots for neutralino dark matter, JHEP 05 (2013) 100 [arXiv:1211.4873] [INSPIRE].

[41] M.E. Cabrera, J.A. Casas and B. Zaldívar, New techniques for chargino-neutralino detection at LHC, JHEP 08 (2013) 058 [arXiv:1212.5247] [INSPIRE].

[42] H. Baer et al., Same sign diboson signature from supersymmetry models with light higgsinos at the LHC, Phys. Rev. Lett. 110 (2013) 151801 [arXiv:1302.5816] [INSPIRE].

[43] A.G. Delannoy et al., Probing dark matter at the LHC using vector boson fusion processes, Phys. Rev. Lett. 111 (2013) 061801 [arXiv:1304.7779] [InSPIRE].

[44] S. Gori, S. Jung and L.-T. Wang, Cornering electroweakinos at the LHC, JHEP 10 (2013) 191 [arXiv: 1307.5952] [INSPIRE].

[45] T. Han, S. Padhi and S. Su, Electroweakinos in the light of the Higgs boson, Phys. Rev. D 88 (2013) 115010 [arXiv:1309.5966] [INSPIRE].

[46] C. Han et al., Probing light higgsinos in natural SUSY from monojet signals at the LHC, JHEP 02 (2014) 049 [arXiv:1310.4274] [INSPIRE].

[47] P. Schwaller and J. Zurita, Compressed electroweakino spectra at the LHC, JHEP 03 (2014) 060 [arXiv: 1312.7350] [INSPIRE].

[48] H. Baer, A. Mustafayev and X. Tata, Monojets and mono-photons from light higgsino pair production at LHC14, Phys. Rev. D 89 (2014) 055007 [arXiv:1401.1162] [INSPIRE].

[49] Z. Han, G.D. Kribs, A. Martin and A. Menon, Hunting quasidegenerate higgsinos, Phys. Rev. D 89 (2014) 075007 [arXiv: 1401.1235] [INSPIRE].

[50] A. Anandakrishnan, L.M. Carpenter and S. Raby, Degenerate gaugino mass region and mono-boson collider signatures, Phys. Rev. D 90 (2014) 055004 [arXiv:1407.1833] [INSPIRE].

[51] J. Bramante, A. Delgado, F. Elahi, A. Martin and B. Ostdiek, Catching sparks from well-forged neutralinos, Phys. Rev. D 90 (2014) 095008 [arXiv:1408.6530] [INSPIRE].

[52] C. Han, L. Wu, J.M. Yang, M. Zhang and Y. Zhang, A new approach for detecting compressed bino/wino at the LHC, arXiv:1409.4533 [INSPIRE]. 
[53] H. Baer, A. Mustafayev, E.-K. Park and X. Tata, Collider signals and neutralino dark matter detection in relic-density-consistent models without universality, JHEP 05 (2008) 058 [arXiv:0802.3384] [INSPIRE].

[54] G. Bélanger, S. Biswas, C. Boehm and B. Mukhopadhyaya, Light neutralino dark matter in the MSSM and its implication for LHC searches for staus, JHEP 12 (2012) 076 [arXiv:1206.5404] [INSPIRE].

[55] J. Liu, B. Shuve, N. Weiner and I. Yavin, Looking for new charged states at the LHC: signatures of magnetic and Rayleigh dark matter, JHEP 07 (2013) 144 [arXiv:1303.4404] [INSPIRE].

[56] L. Calibbi, J.M. Lindert, T. Ota and Y. Takanishi, Cornering light neutralino dark matter at the LHC, JHEP 10 (2013) 132 [arXiv: 1307.4119] [INSPIRE].

[57] M. Cirelli, F. Sala and M. Taoso, Wino-like minimal dark matter and future colliders, JHEP 10 (2014) 033 [arXiv: 1407.7058] [InSPIRE].

[58] A. Pierce, N.R. Shah and K. Freese, Neutralino dark matter with light staus, arXiv: 1309.7351 [INSPIRE].

[59] M. Low and L.-T. Wang, Neutralino dark matter at $14 \mathrm{TeV}$ and $100 \mathrm{TeV}$, JHEP 08 (2014) 161 [arXiv: 1404.0682] [INSPIRE].

[60] C. Boehm, P.S.B. Dev, A. Mazumdar and E. Pukartas, Naturalness of light neutralino dark matter in pMSSM after LHC, XENON100 and Planck data, JHEP 06 (2013) 113 [arXiv:1303.5386] [INSPIRE].

[61] M.W. Cahill-Rowley, J.L. Hewett, S. Hoeche, A. Ismail and T.G. Rizzo, The new look pMSSM with neutralino and gravitino LSPs, Eur. Phys. J. C 72 (2012) 2156 [arXiv:1206.4321] [INSPIRE].

[62] M. Cahill-Rowley, J.L. Hewett, A. Ismail and T.G. Rizzo, pMSSM studies at the 7, 8 and $14 \mathrm{TeV} L H C$, arXiv:1307.8444 [INSPIRE].

[63] J. Alwall, M. Herquet, F. Maltoni, O. Mattelaer and T. Stelzer, MadGraph 5: going beyond, JHEP 06 (2011) 128 [arXiv:1106.0522] [INSPIRE].

[64] W. Beenakker, R. Hopker and M. Spira, PROSPINO: a program for the production of supersymmetric particles in next-to-leading order QCD, hep-ph/9611232 [INSPIRE].

[65] W. Beenakker et al., The production of charginos/neutralinos and sleptons at hadron colliders, Phys. Rev. Lett. 83 (1999) 3780 [Erratum ibid. 100 (2008) 029901] [hep-ph/9906298] [INSPIRE].

[66] A. Djouadi, M.M. Muhlleitner and M. Spira, Decays of supersymmetric particles: the program SUSY-HIT (SUspect-SdecaY-HDECAY-InTerface), Acta Phys. Polon. B 38 (2007) 635 [hep-ph/0609292] [INSPIRE].

[67] A. Djouadi, J.-L. Kneur and G. Moultaka, SuSpect: a fortran code for the supersymmetric and Higgs particle spectrum in the MSSM, Comput. Phys. Commun. 176 (2007) 426 [hep-ph/0211331] [INSPIRE].

[68] B.C. Allanach, SOFTSUSY: a program for calculating supersymmetric spectra, Comput. Phys. Commun. 143 (2002) 305 [hep-ph/0104145] [InSPIRE]. 
[69] J.M. Cornwall, D.N. Levin and G. Tiktopoulos, Derivation of gauge invariance from high-energy unitarity bounds on the s matrix, Phys. Rev. D 10 (1974) 1145 [Erratum ibid. D 11 (1975) 972] [INSPIRE].

[70] C.E. Vayonakis, Born helicity amplitudes and cross-sections in nonabelian gauge theories, Lett. Nuovo Cim. 17 (1976) 383 [INSPIRE].

[71] B.W. Lee, C. Quigg and H.B. Thacker, Weak interactions at very high-energies: the role of the Higgs boson mass, Phys. Rev. D 16 (1977) 1519 [InSPIRE].

[72] C. Cheung, J.T. Ruderman, L.-T. Wang and I. Yavin, Lepton jets in (supersymmetric) electroweak processes, JHEP 04 (2010) 116 [arXiv:0909.0290] [INSPIRE].

[73] T. Sjöstrand, S. Mrenna and P.Z. Skands, PYTHIA 6.4 physics and manual, JHEP 05 (2006) 026 [hep-ph/0603175] [INSPIRE].

[74] J. Alwall, S. de Visscher and F. Maltoni, QCD radiation in the production of heavy colored particles at the LHC, JHEP 02 (2009) 017 [arXiv: 0810.5350] [INSPIRE].

[75] DELPHES 3 collaboration, J. de Favereau et al., DELPHES 3, a modular framework for fast simulation of a generic collider experiment, JHEP 02 (2014) 057 [arXiv:1307.6346] [INSPIRE].

[76] ATLAS collaboration, Performance assumptions for an upgraded ATLAS detector at a high-luminosity LHC, ATL-PHYS-PUB-2013-004, CERN, Geneva Switzerland (2013).

[77] D. Whiteson, private correspondance, December 122013.

[78] C.G. Lester and D.J. Summers, Measuring masses of semiinvisibly decaying particles pair produced at hadron colliders, Phys. Lett. B 463 (1999) 99 [hep-ph/9906349] [INSPIRE].

[79] A. Barr, C. Lester and P. Stephens, $m_{T 2}$ : the truth behind the glamour, J. Phys. G 29 (2003) 2343 [hep-ph/0304226] [INSPIRE].

[80] C. Rogan, Kinematical variables towards new dynamics at the LHC, arXiv:1006.2727 [INSPIRE].

[81] T. Junk, Confidence level computation for combining searches with small statistics, Nucl. Instrum. Meth. A 434 (1999) 435 [hep-ex/9902006] [INSPIRE].

[82] L. Moneta et al., The RooStats project, PoS (ACAT2010) 057 [arXiv: 1009.1003] [INSPIRE].

[83] ATLAS collaboration, Search for direct production of charginos and neutralinos in events with three leptons and missing transverse momentum in $\sqrt{s}=8 \mathrm{TeV}$ pp collisions with the ATLAS detector, JHEP 04 (2014) 169 [arXiv: 1402.7029] [INSPIRE].

[84] ATLAS collaboration, Search for supersymmetry in events with four or more leptons in $21 \mathrm{fb}^{-1}$ of pp collisions at $\sqrt{s}=8 \mathrm{TeV}$ with the ATLAS detector, ATLAS-CONF-2013-036, CERN, Geneva Switzerland (2013).

[85] ATLAS collaboration, Search for strongly produced supersymmetric particles in decays with two leptons at $\sqrt{s}=8 \mathrm{TeV}$, ATLAS-CONF-2013-089, CERN, Geneva Switzerland (2013).

[86] ATLAS collaboration, Search for electroweak production of supersymmetric particles in final states with at least two hadronically decaying taus and missing transverse momentum with the ATLAS detector in proton-proton collisions at $\sqrt{s}=8 \mathrm{TeV}$,

ATLAS-CONF-2013-028, CERN, Geneva Switzerland (2013). 
[87] ATLAS collaboration, Search for strongly produced superpartners in final states with two same sign leptons with the ATLAS detector using $21 \mathrm{fb}^{-1}$ of proton-proton collisions at $\sqrt{s}=8 \mathrm{TeV}$., ATLAS-CONF-2013-007, CERN, Geneva Switzerland (2013).

[88] ATLAS collaboration, Search for new phenomena in monojet plus missing transverse momentum final states using $10 \mathrm{fb}^{-1}$ of pp collisions at $\sqrt{s}=8 \mathrm{TeV}$ with the ATLAS detector at the LHC, ATLAS-CONF-2012-147, CERN, Geneva Switzerland (2012).

[89] ATLAS collaboration, Search for squarks and gluinos with the ATLAS detector in final states with jets and missing transverse momentum and $20.3 \mathrm{fb}^{-1}$ of $\sqrt{s}=8 \mathrm{TeV}$ proton-proton collision data, ATLAS-CONF-2013-047, CERN, Geneva Switzerland (2013).

[90] ATLAS collaboration, Search for charginos nearly mass degenerate with the lightest neutralino based on a disappearing-track signature in pp collisions at $\sqrt{s}=8 \mathrm{TeV}$ with the ATLAS detector, Phys. Rev. D 88 (2013) 112006 [arXiv:1310.3675] [INSPIRE].

[91] CMS collaboration, Search for electroweak production of charginos, neutralinos and sleptons using leptonic final states in pp collisions at $8 \mathrm{TeV}$, CMS-PAS-SUS-13-006, CERN, Geneva Switzerland (2013).

[92] CMS collaboration, Search for new physics in monojet events in pp collisions at $\sqrt{s}=8 \mathrm{TeV}$, CMS-PAS-EXO-12-048, CERN, Geneva Switzerland (2012).

[93] LEP2 SUSY WorkING GRouP collaboration, Combined LEP chargino results, up to $208 \mathrm{GeV}$ for large $m 0$ webpage, http://lepsusy.web.cern.ch/lepsusy/www/ inos_moriond01/charginos_pub.html.

[94] LEP2 SUSY Working GRouP collaboration, Combined LEP chargino results, up to 208 GeV for low DM webpage, http://lepsusy.web.cern.ch/lepsusy/www/ inoslowdmsummer02/charginolowdm_pub.html.

[95] A. Rajaraman, W. Shepherd, T.M.P. Tait and A.M. Wijangco, LHC bounds on interactions of dark matter, Phys. Rev. D 84 (2011) 095013 [arXiv: 1108.1196] [InSPIRE].

[96] P.J. Fox, R. Harnik, J. Kopp and Y. Tsai, Missing energy signatures of dark matter at the LHC, Phys. Rev. D 85 (2012) 056011 [arXiv:1109.4398] [InSPIRE].

[97] P.J. Fox, R. Harnik, R. Primulando and C.-T. Yu, Taking a razor to dark matter parameter space at the LHC, Phys. Rev. D 86 (2012) 015010 [arXiv:1203.1662] [InSPIRE].

[98] M.R. Buckley, J.D. Lykken, C. Rogan and M. Spiropulu, Super-razor and searches for sleptons and charginos at the LHC, Phys. Rev. D 89 (2014) 055020 [arXiv:1310.4827] [INSPIRE].

[99] L. Randall and R. Sundrum, Out of this world supersymmetry breaking, Nucl. Phys. B 557 (1999) 79 [hep-th/9810155] [INSPIRE].

[100] G.F. Giudice, M.A. Luty, H. Murayama and R. Rattazzi, Gaugino mass without singlets, JHEP 12 (1998) 027 [hep-ph/9810442] [INSPIRE].

[101] M. Ibe, S. Matsumoto and R. Sato, Mass splitting between charged and neutral winos at two-loop level, Phys. Lett. B 721 (2013) 252 [arXiv:1212.5989] [InSPIRE].

[102] J.L. Feng, T. Moroi, L. Randall, M. Strassler and S.-F. Su, Discovering supersymmetry at the Tevatron in wino LSP scenarios, Phys. Rev. Lett. 83 (1999) 1731 [hep-ph/9904250] [INSPIRE]. 
[103] T. Gherghetta, G.F. Giudice and J.D. Wells, Phenomenological consequences of supersymmetry with anomaly induced masses, Nucl. Phys. B 559 (1999) 27 [hep-ph/9904378] [INSPIRE].

[104] G.D. Kribs, A. Martin and T.S. Roy, Supersymmetry with a chargino NLSP and gravitino LSP, JHEP 01 (2009) 023 [arXiv:0807.4936] [INSPIRE].

[105] H.K. Dreiner, M. Krämer and J. Tattersall, How low can SUSY go? Matching, monojets and compressed spectra, Europhys. Lett. 99 (2012) 61001 [arXiv:1207.1613] [INSPIRE].

[106] S. Mukhopadhyay, M.M. Nojiri and T.T. Yanagida, Compressed SUSY search at the $13 \mathrm{TeV}$ LHC using kinematic correlations and structure of ISR jets, JHEP 10 (2014) 012 [arXiv: 1403.6028] [INSPIRE].

[107] J.M. Cline, M. Joyce and K. Kainulainen, Supersymmetric electroweak baryogenesis, JHEP 07 (2000) 018 [hep-ph/0006119] [INSPIRE].

[108] M.S. Carena, J.M. Moreno, M. Quirós, M. Seco and C.E.M. Wagner, Supersymmetric CP-violating currents and electroweak baryogenesis, Nucl. Phys. B 599 (2001) 158 [hep-ph/0011055] [INSPIRE].

[109] Y. Li, S. Profumo and M. Ramsey-Musolf, Bino-driven electroweak baryogenesis with highly suppressed electric dipole moments, Phys. Lett. B 673 (2009) 95 [arXiv:0811.1987] [INSPIRE].

[110] H.E. Haber and G.L. Kane, The search for supersymmetry: probing physics beyond the standard model, Phys. Rept. 117 (1985) 75 [INSPIRE].

[111] S.P. Martin, A supersymmetry primer, Adv. Ser. Direct. High Energy Phys. 21 (2010) 1 [hep-ph/9709356] [INSPIRE].

[112] D.J.H. Chung et al., The soft supersymmetry breaking Lagrangian: theory and applications, Phys. Rept. 407 (2005) 1 [hep-ph/0312378] [INSPIRE].

[113] J.F. Gunion, H.E. Haber, G.L. Kane and S. Dawson, The Higgs hunter's guide, Front. Phys. 80 (2000) 1 [inSPIRE].

[114] J.F. Gunion, H.E. Haber, G.L. Kane and S. Dawson, Errata for the Higgs hunter's guide, hep-ph/9302272 [INSPIRE]. 